TITLE:

\title{
Non-contact R-test with laser displacement sensors for error calibration of five-axis machine tools
}

\author{
AUTHOR(S): \\ Hong, Cefu; Ibaraki, Soichi
}

\section{CITATION:}

Hong, Cefu ... [et al]. Non-contact R-test with laser displacement sensors for error calibration of five-axis machine tools. Precision Engineering 2013, 37(1): 159-171

\section{ISSUE DATE:}

2013-01

URL:

http://hdl.handle.net/2433/162006

\section{RIGHT:}

(c) 2012 Elsevier Inc.; This is not the published version. Please cite only the published version.; この論文は出版社版でありません。引用の際に は出版社版をご確認ご利用ください。 
Non-contact R-test with laser displacement sensors for error calibration of five-axis machine tools

Cefu Hong ${ }^{1}$, Soichi Ibaraki ${ }^{1 \#}$

1 Department of Micro Engineering, Graduate School of Engineering, Kyoto University, Yoshida-honmachi, Sakyo-ku, Kyoto 606-8501, Japan

\# Corresponding Author / E-mail: ibaraki@prec.kyoto-u.ac.jp, TEL: +81-75-753-5227,

FAX: $+81-75-753-5227$

\section{Abstract:}

The R-test is an instrument to measure three-dimensional displacement of a precision sphere attached to a spindle relative to a work table by using three displacement sensors. Its application to error calibration for five-axis machine tools has been studied in both academia and industry. For the simplicity in calculating the sphere center displacement, all conventional R-test devices use contact-type displacement sensors with a flat-ended probe. Conventional contact-type R-test may be potentially subject to the influence of the friction or the dynamics of supporting spring in displacement sensors particularly in dynamic measurement. This paper proposes a non-contact R-test with laser displacement sensors. First, a new algorithm is proposed to calculate the three-dimensional displacement of sphere center by using non-contact displacement sensors. The compensation of measurement error of a laser displacement sensor due to the curvature of target sphere is incorporated. Then, the measurement uncertainty of four laser displacement sensors with different measuring principles is experimentally investigated in measuring the geometry of a sphere in order to select the laser displacement sensor most suitable for the application to a non-contact R-test. A prototype non-contact R-test device is developed for the verification of the proposed algorithm for non-contact R-test. Experimental case studies of error calibration of 1) static 
and 2) dynamic error motions of rotary axes in a five-axis machine tool with the developed non-contact R-test prototype are presented. Its measurement performance is compared to the conventional contact-type R-test device.

Keywords: R-test, error calibration, dynamic measurement, five-axis machine tool, laser displacement sensor

\section{Introduction}

Machine tools with two rotary axes to tilt and rotate a tool with respect to a workpiece, in addition to three orthogonal linear axes, are collectively called five-axis machine tools. With an increasing need for machining components with geometric complexity in a high efficiency, they are extensively used in various manufacturing applications. The improvement of their motion accuracy is a crucial demand in the market. As its basis, it is important to develop a methodology to measure it in an accurate and efficient manner. Many measurement schemes for error calibration of five-axis machine tools have been recently studied in the literature, as reviewed in [1,2].

The R-test, first proposed by Weikert [3], is a new instrument to measure three-dimensional displacement of a precision sphere attached to a spindle relative to a work table by using three displacement sensors. The R-test, or the radial test, is described in recently revised ISO 230-1:2012 (Clause 11.3.5.3) [4]. The inclusion of R-test application to dynamic measurement of synchronization error of a rotary axis to linear axes in the revised ISO 10791-6 [5] has been under discussion in ISO/TC39/SC2. Lately, IBS Precision Engineering [6] and Fidia [7] commercialized an R-test device for machine tool calibration. Bringmann and Knapp [8] presented its application to the identification of location errors of rotary axes for five-axis machine tools. Location errors of a rotary axis represent the 
position and the orientation of its axis average line, i.e. the straight line representing the mean location and orientation of its axis of rotation [9], and can be seen as one of the most fundamental error sources in the five-axis kinematics. Ibaraki et al. [10,11] have proposed its extension to construct an error map (or 'component errors' defined in [9]) of rotary axes. Dynamic R-test measurements have been presented in $[12,13]$.

All the conventional R-test devices, including commercially available ones [6,7], use a contact-type linear displacement sensor with a flat-ended probe (the one by IBS Precision Engineering [6] uses non-contact displacement sensors, but it is the same in that a flat-ended probe touches the sphere). When flat-ended probes are contacted with the sphere, its three-dimensional displacement can be calculated by a simple formula $[3,8]$ from measured displacements by using pre-calibrated direction vectors of linear displacement sensors only. However, the influence of friction between probes and the sphere, or the dynamics of a spring supporting probes, could potentially impose significant influence on the measured displacement, particularly in dynamic measurements. Furthermore, the safety of measurement, avoiding the crash caused by e.g. mis-programming, can be a critical issue with the contact-type R-test. Therefore, a non-contact R-test has potentially significant advantages particularly for dynamic measurement on five-axis machine tool.

This paper proposes a non-contact type R-test with laser displacement sensors. Potential inherent advantages of applying non-contact displacement sensors to the R-test include: 1) the measurement is not affected at all by the friction on the sphere surface and probes; 2) the measurement is not affected at all by the dynamics of a supporting spring in contact-type displacement sensors; 3) the measurement is safer due to longer working distance between the sphere and sensors. As a non-contact displacement sensor, we employ a laser displacement sensor for its longer reference distance and larger measurement range than other non-contact sensors, such as capacitive and inductive displacement sensors. 
For conventional contact-type R-test devices, the influence of friction or supporting springs may significantly vary due to measurement conditions (e.g. feed rate) or the material property of sphere or probe surface. Although it is difficult to quantitatively evaluate the measurement uncertainty attributable to the friction or spring dynamics in general cases, test results presented in this paper (Section 5.2, Figs. 21 to 23) suggest that our conventional contact-type R-test prototype is subject to the measurement uncertainty up to about $6 \mu \mathrm{m}$ in dynamic tests. Considering typical motion accuracy of commercial small- or medium-sized five-axis machining centers, as well as tolerances for dynamic R-tests described in ISO/DIS 10791-6 [5], our goal is to develop a prototype non-contact R-test device of the measurement uncertainty about $1 \mu \mathrm{m}$ in dynamic tests. Furthermore, the prototype is designed such that the measurable volume of sphere's relative displacement is larger than $1 \mathrm{~mm} \times 1 \mathrm{~mm} \times 1 \mathrm{~mm}$. Due to e.g. mis-calibration of rotary axis position, the sphere's relative displacement in R-test cycles could be as large as several hundred micrometers.

When a non-contact displacement sensor is used, or when the sensor does not touch the sphere with a flat surface, e.g. sphere-ended probes, the offset of the sphere center from the line representing the sensor's sensitive direction could introduce an error for calculating the three-dimensional displacement of the sphere when the conventional algorithm for contact-type R-test is used [14]. A new algorithm for calculating three-dimensional displacement of sphere with non-contact displacement sensors should be proposed. Note that Zargarbashi and Mayer [15] have proposed an analogous non-contact R-test device, named 'Cap-ball', with capacitance displacement sensors. Their algorithm used for calculating the three-dimensional displacement of sphere seemed, however, the same with the algorithm used in the conventional R-test. For non-contact sensors, the conventional algorithm is valid only when the measuring range of each sensor is sufficiently small. Another critical issue with the application of a laser displacement sensor to the non-contact 
R-test is its measurement uncertainty due to sphere curvature (i.e. the inclination of the target surface). When the sensor's sensitive direction is off the center of the sphere, the target surface, where the laser spot hits, is inclined from the sensor's sensitive direction, which often results in the measurement error. An appropriate laser displacement sensor should be chosen, whose measurement uncertainty is less sensitive to sphere curvature. Furthermore, such a measurement error must be numerically compensated from measured displacement profiles.

To develop a prototype non-contact R-test device, this paper experimentally investigates the measurement uncertainty in measuring spherical surface of four laser displacement sensors available in today's market, in order to select the laser displacement sensor most suitable for non-contact R-test. The investigated laser displacement sensors include spectral interference type (SI-F10 by Keyence), specular reflection type (LK-G10 by Keyence), diffuse reflection type (LK-H052 by Keyence), and confocal type (LT-9010MS by Keyence) [16].

A prototype non-contact R-test device with the specular reflection type laser displacement sensor (LK-G10 by Keyence) is developed for the experimental verification of the proposed algorithm for non-contact R-test. Experimental case studies of error calibration on a five-axis machine tool, including static and dynamic measurement by the developed non-contact R-test device, are presented to evaluate its measurement performance by comparing with the contact-type R-test device.

2. Algorithm to calculate three-dimensional displacement of sphere center by non-contact R-test

2.1 Algorithm for conventional contact-type R-test

Figure 1 shows the schematics of the conventional contact-type R-test device used in [10, 11]. A precision sphere is attached to a machine spindle. Three contact-type linear 
displacement sensors $([3,8]$ used four sensors) with a flat-ended probe are attached on a fixture (named "sensors nest" [5]) that is fixed on a rotary table. This subsection briefly reviews the algorithm to calculate the sphere center displacement by this contact-type R-test $[3,8]$.

A flat-ended probe is pushed to and contacted with the sphere. Therefore, its displacement would not be affected by center offset of the sphere in the plane perpendicular to the sensor's sensitive direction (see Fig. 2 (a)).

Figure 3 shows the measurement setup of conventional contact-type R-test (only one displacement sensor is shown). The origin of the coordinate system, $O_{0}=(0,0,0)^{\mathrm{T}}$, is defined at the sphere center in its initial position. The unit direction vector $V_{i}=\left(u_{i}, v_{i}, w_{i}\right)^{\mathrm{T}}(i$ $=1,2,3)$ represents the sensitive direction of the $i$-th displacement sensor. The displacement of sphere center, $O_{j}=\left(O_{j x}, O_{j y}, O_{j z}\right)^{\mathrm{T}}(j=1, \ldots, N)$, can be calculated as follows:

$$
\left[\begin{array}{lll}
O_{j x} & O_{j y} & O_{j z}
\end{array}\right]=\left[\begin{array}{lll}
d_{1 j} & d_{2 j} & d_{3 j}
\end{array}\right] \cdot\left[\begin{array}{ccc}
u_{1} & u_{2} & u_{3} \\
v_{1} & v_{2} & v_{3} \\
w_{1} & w_{2} & w_{3}
\end{array}\right]^{-1}
$$

where $d_{i j}(i=1,2,3 ; j=1, \ldots, N)$ denotes the measured displacement of the $i$-th displacement sensor when the sphere center is moved from $O_{0}$ to $O_{j}$. It suggests that when three displacement sensors touch the sphere from different directions, the three-dimensional displacement of the sphere center can be calculated from pre-calibrated direction vectors and measured displacements of three probes only.

\subsection{Algorithm for non-contact R-test}

For a non-contact R-test, the measured displacement of each probe changes when the sphere center is shifted perpendicular to the sensitive direction of laser displacement sensor (see 
Fig. 2(b)). It suggests that an error would be introduced when the conventional algorithm for contact-type R-test is used. The objective of this subsection is to propose a new algorithm for calculating three-dimensional displacement of sphere with non-contact displacement sensors.

Figure 4 shows the measurement setup of a non-contact R-test. A precision sphere with the radius $R$ is attached to the spindle of test machine. Three laser displacement sensors $(i=1,2$, 3), which are installed on the table, are directed to the sphere center only roughly. The origin of the coordinate system, $O_{0}=(0,0,0)^{\mathrm{T}}$, is defined at the sphere center in its initial position; the orientation of the coordinate system is defined based on the machine tool coordinate system [3]. The unit direction vector $V_{i}=\left(u_{i}, v_{i}, w_{i}\right)^{\mathrm{T}}(i=1,2,3)$ represents the sensitive measurement direction of the $i$-th laser displacement sensor. The intersection point of the line representing $i$-th sensor's sensitive direction and the sphere surface at the initial position is defined as $P_{i 0}=\left(x_{i 0}, y_{i 0}, z_{i 0}\right)^{\mathrm{T}}(i=1,2,3)$. When the sphere center is positioned at $O_{j}=\left(O_{j x}, O_{j y}, O_{j z}\right)^{\mathrm{T}}(j=1, \ldots, N)$, the intersection moves to $P_{i j}=\left(x_{i j}, y_{i j}, z_{i j}\right)^{\mathrm{T}}(i=1,2,3) . d_{i j}$ $(i=1,2,3 ; j=1, \ldots, N)$ denotes the measured displacement of the $i$-th laser displacement sensor when the sphere center is moved from $O_{0}$ to $O_{j}$.

The relationship between the displacement of laser displacement sensors and the position of the sphere center is represented by:

$$
\left\{\begin{array}{l}
P_{i j}=P_{i 0}+d_{i j} V_{i} \\
\left\|P_{i j}-O_{j}\right\|^{2}=R^{2}
\end{array}\right.
$$

The procedure to calculate the three-dimensional position of the sphere center, $O_{j}$, with non-contact R-test is shown as follows:

(1) Calibration of parameters of each sensor

Similarly as the conventional contact-type R-test, the direction vector of each sensor, $V_{i}(i=$ 
1, 2, 3), must be pre-calibrated in the machine tool coordinate system. However, the estimation of the sphere center, $O_{j}$, from laser displacements, $d_{i j}(i=1,2,3)$, requires not only the orientation of each sensor's sensitive direction, $V_{i}$, but also intersection points when the sphere is at the initial position, represented by $P_{i 0}(i=1,2,3)$. This is an essential difference in algorithms for contact-type and non-contact R-tests. Their calibration procedure of parameters mentioned above is shown as follows:

a. Position the sphere center at the given set of reference positions, $O_{j}(j=1, \ldots, N)$, within the measurement volume;

b. Assuming that the machine's positioning error at $O_{j}$ is sufficiently small compared to the nominal moving distance, calibrate $P_{i 0}$ and $V_{i}(i=1,2,3)$, from $O_{j}(j=1, \ldots, N)$ and measured laser displacements $d_{i j}(j=1, \ldots, N)$ by solving the following minimization problem:

$$
\min _{P_{i 0}, V_{i}} \sum_{j=1, \ldots, N_{1}}\left(\left\|P_{i 0}+d_{i j} \cdot V_{i}-O_{j}\right\|-R\right)^{2}
$$

where equality constraints $\left\|P_{i 0}\right\|=R,\left\|V_{i}\right\|=1(i=1,2,3)$ are applied.

(2) Estimation of sphere center displacement from laser displacements

Once the direction vector of each sensor, $V_{i}(i=1,2,3)$, as well as its position, represented by $P_{i 0}(i=1,2,3)$, is calibrated, the three-dimensional displacement of sphere center, $O_{j}(j$ $\left.=1, \ldots, N_{2}\right)$, can be calculated from measured laser displacements, $d_{i j}(i=1,2,3)$, by solving the following minimization problem:

$$
\min _{O_{j}} \sum_{i=1,2,3}\left(\left\|P_{i 0}+d_{i j} \cdot V_{i}-O_{j}\right\|-R\right)^{2}
$$

Both Eq. (3) and Eq. (4) can be locally solved with a nonlinear least square method [17].

2.3 Compensation scheme of measurement error for non-contact R-test

Laser displacement sensors generally exhibit the best measurement performance when the 
measured surface is placed normal to the sensor's sensitive direction. Therefore, when measuring a sphere surface, the measurement uncertainty is supposedly minimized when the laser is directed exactly to the sphere center. When the sphere center is shifted from there, the measured surface becomes tilted from the sensor's sensitive direction, and as a result, the measurement uncertainty is expected to increase. Figure 5 shows the specular reflection type laser displacement sensor as an example, but similar observation can be made for other sensors. As will be shown in Section 3.1, this measurement error cannot be ignored for the required measurement accuracy of the non-contact R-test. This subsection proposes a scheme to compensate this measurement error. This measurement error is assumed to be quasi-static, i.e. the error depends only on the sphere center position relative to the laser displacement sensors.

We employ a radial basis function (RBF) neural network as a three-dimensional look-up table to interpolate the measurement error of laser displacement sensors. In the RBF network [18], a weight matrix $w_{i j}(\hat{x}(i)) \in \mathfrak{R}^{3}(j=1, \ldots, \mathrm{N})$ and radial basis functions $\varphi_{i j}(\hat{x}(i)) \in \mathfrak{R}^{3}$ are together used to describe the measurement error $b_{i}(\hat{x}(i)) \in \mathfrak{R}^{3} \quad(i=$ $1, \ldots, M)$ as in Eq. (5). Note that $\hat{x}(i) \in \mathfrak{R}^{3}$ represents the 'rough' estimate of the $i$-th sphere center position by the algorithm proposed in the previous subsection, and is the input to the RBF network. The radial basis function, as shown in Eq. (6), represents the three-dimensional displacement of the estimated sphere center position $\hat{x}(i)$ from another position, $\hat{x}(j)(j=1, \ldots, \mathrm{N})$, in the measurement volume.

$$
\begin{gathered}
\sum_{j=1}^{N} \varphi_{i j}(\hat{x}(i)) w_{i j}(\hat{x}(i))=b_{i}(\hat{x}(i))(i=1, \ldots, M) \\
\varphi_{i j}(\hat{x}(i))=\hat{x}(j)-\hat{x}(i)(j=1, \ldots, N)
\end{gathered}
$$

The compensated sphere displacement is given by: 


$$
x_{\text {comp }}(i)=\hat{x}(i)-b(\hat{x}(i))
$$

In the RBF network, weight matrices, $w_{i j}(\hat{x}(i))$, are trained by a calibration procedure analogous to the one in Section $2.2(1)$.

3. Experimental investigation of performance of laser displacement sensors for measuring spherical surface

\subsection{Objective}

Many laser displacement sensors with different measuring principle are available in today's market. To give guidance to the selection of a laser displacement sensor for a prototype non-contact R-test device, this section experimentally investigates the measurement uncertainty of four laser displacement sensors in spherical surface measurement.

For the application to a non-contact R-test, it is crucial to select an appropriate type of laser displacement sensor with the following properties:

(1) The measurement uncertainty caused by the inclination of the target surface is smaller.

(2) High frequency measurement "noise", typically caused by the speckle noise in the laser beam, is smaller.

(3) Larger "measurable area" on a sphere surface can be obtained. In this section, "measurable area" represents the area on the spherical surface where valid reflected laser beam returns to the sensor and the measurement is possible.

(4) The measurement range, defined as the working distance along the sensor's sensitive direction, is larger.

To find the best laser displacement sensor for the application to a non-contact R-test, we experimentally investigate the sensitivity of measuring uncertainty to the sphere curvature by a profile measurement of sphere surface. 


\subsection{Laser displacement sensors}

The following four commercial laser displacement sensors of different measuring principle, as schematically illustrated in Figs. 6 (a) to (d), are compared. Table 1 shows main specifications of the investigated laser displacement sensors. Their measuring principles are briefly introduced as follows (more detailed information can be found in [16]):

(a) Specular reflection type (LK-G10 by Keyence, Fig. 6(a)).

(b) Diffuse reflection type (LK-H052 by Keyence, Fig. 6(b)).

These laser displacement sensors focus the laser beam on a target. The target reflects the beam back through the lens where it is focused on a light-receiving element, forming a beam spot. By detecting the movement of the beam spot, the target's displacement can be determined. The surface-reflected laser from a target object consists of a regular reflection and a diffuse reflection component. Sensors (a) and (b) accept regular reflection component and diffuse reflection component, respectively.

(c) Spectral interference type (SI-F10 by Keyence, Fig. 6(c)).

A target-reflected laser beam with wide wavelength band, interferes with the laser beam from a reference surface. The spectrum of the interfered laser beam is detected by a light-receiving element and analyzed to determine the displacement of the target.

(d) Confocal type (LT-9010MS by Keyence, Fig. 6(d)).

A laser beam is focused on a target surface through an objective lens that vibrates up and down at high speed by means of a tuning fork. The beam reflected off the target surface is converged on a pinhole and then enters a light-receiving element. By measuring the exact position of the objective lens when the light enters the receiving element, the target height can be determined. 


\subsection{Test procedure}

First, a ceramic precision sphere with the radius of $12.7 \mathrm{~mm}$ is attached to the machine spindle. Table 2 shows specifications of the precision sphere. Each of four laser displacement sensors in Table 1 is fixed on the machine table vertically. In each test, the sensor is set up such that the position $(X, Y)=(0,0)$ corresponds to the point where the sensor's sensitive direction approximately points to the sphere center.

Then, the spindle-side sphere is moved in horizontal XY plane to let the laser displacement sensor scan the sphere surface, where the laser displacement is continuously logged. Similar scanning test is conducted for each sensor, although the scanning direction, the scanning range, and the scanning path pitch differ for each sensor.

When the sensor's sensitive direction is exactly pointing to the sphere center at $(X, Y)=(0$, 0), the measured laser displacement should be the shortest. Due to its setup error, this "top" location, as well as the orientation of measured profile, may be shifted from the nominal position and orientation. To cancel the influence of this setup error, the ideal sphere profile is best-fit to the measured profile. The difference of measured profiles from the best-fit ideal sphere is then recalculated. The profile of the measured sphere surface is generated with nominal X-and Y-positions of the sensor.

\subsection{Experimental results and observations}

(1) Experimental results

Figures 7-10 show the profile measurement result by each laser displacement sensor. In Figs. $7-10$ (a), the nominal sphere profile of the radius $12.7 \mathrm{~mm}$ is best-fit to the measured profile. In Figs. 7-10(b), for each of scanning line to the X-direction, an error of the measured profile from the nominal profile is plotted (all the scanning lines are superimposed). This profile is called the profile measurement error. 
(2) Observations

The following observations can be made for each sensor:

(1) Specular reflection type (LK-G10, Fig. 7)

In this test, within the range $\pm 0.5 \mathrm{~mm}$ from the sphere top, the average error from the sphere's nominal geometry is within $\pm 3 \mu \mathrm{m}$ approximately. The higher-frequency "noise" in measured profiles of the amplitude up to $2 \mu \mathrm{m}$ is also observed over the entire range, which is likely caused by the speckle noise in laser beam.

(2) Diffuse reflection type (LK-H052, Fig. 8)

Diffuse reflection type laser displacement sensor (LK-H052 from Keyence) succeeded to measure the widest range of sphere surface (over $\pm 2.5 \mathrm{~mm}$ ) among the four sensors, while higher-frequency "noise" is significantly larger (its amplitude is up to $15 \mu \mathrm{m}$ ) than other laser displacement sensors, which could be caused by the speckle noise in laser beam. More studies on the measurement uncertainty of this sensor for profile measurement can be found in [19].

(3) Spectral interference type (SI-F10, Fig. 9)

Although this sensor has smaller measurement range $( \pm 0.3 \mathrm{~mm})$, the test result in Fig. 9 shows smallest measurement errors (the mean error is within $\pm 0.2 \mu \mathrm{m}$ ). Its higher-frequency component is also the lowest among the four sensors.

(4) Confocal type (LT-9010MS, Fig. 10)

For confocal type laser displacement sensor, both the profile measurement error (within $1 \mu \mathrm{m}$ in the range $\pm 0.5 \mathrm{~mm}$ ) and higher-frequency error (within $\pm 0.8 \mu \mathrm{m}$ ) are relatively small among the four sensors. However, occasional spikes, of the amplitude $6 \mu \mathrm{m}$ at maximum, are observed. This could be caused by the focusing error due to the laser's speckle noise. 
Test results are summarized in Table 3. For quantitative comparison, two types of the measurable area are defined by the distance from the line pointing to the sphere center where:

1) the profile measurement error is smaller than the given tolerance $(1 \mu \mathrm{m})$;

2) the profile measurement error is larger than this tolerance, but the reflected light still returns to the sensor and the measurement is possible.

In Table 3, "profile measurable area" shows the size of the ranges 1) and 2) in the XY plane, experimentally evaluated from test results shown in Figs. 7-10. The noise level is defined as the standard deviation $(1 \sigma)$ of higher-frequency component in the profile measurement error.

From present test results, the specular reflection type laser displacement sensor (LK-G10 by Keyence), which has relatively large measurement range, relatively large measurable area, as well as lower noise level, is chosen as the sensor for an R-test prototype. Although the measurement error due to the target curvature is not sufficiently small over the entire measurable area, it can be compensated as presented in Section 2.3. The cost of each sensor is also one of reasons for this choice. The price of each sensor is: LK-H052 < LK-G10 < LT-9010MS $<$ SI-F10.

\section{Developed prototype non-contact R-test device}

\subsection{Developed prototype}

The developed R-test prototype is shown in Fig. 11. Each sensor is tilted by approximately $45^{\circ}$ from the horizontal plane. A ceramic precision sphere of the diameter $50.0 \mathrm{~mm}$ is attached to the spindle. Table 4 shows major specifications of this precision sphere. Note that, while tests presented in Section 3.3 used a sphere of the diameter $25.4 \mathrm{~mm}$, a sphere of larger diameter is used here for the following reasons: 1) when the $\phi 25.4$ sphere is used, 
three sensors interfere to each other and cannot be mounted as in Fig.11, 2) larger measurable volume of sphere's relative displacement can be obtained.

4.2 Experimental verification of the proposed algorithm to measure sphere center displacement

The objective of this subsection is to experimentally investigate the validity of the algorithm presented in Section 2 to estimate the sphere center position from laser displacements, with the compensation of measurement errors due to the sphere curvature.

The spindle-side sphere is positioned at total 27 positions within a cubic volume $(1 \times 1 \times 1$ $\mathrm{mm}$ ) for parameter calibration presented in Section 2.2 and 2.3. Then, the sphere is positioned at total 1183 positions shown in Fig. 12(a) within the same volume. At each command point, the sphere displacement is estimated from measured laser displacements by the algorithm presented in Section 2.2 and 2.3. As the machine's positioning error is assumed to be sufficiently small, the error between estimated and command positions can be seen as the estimated error of the R-test measurement.

The estimated sphere center position is shown in Fig. 12(a) (the error is magnified by 25 times). The difference in estimated and command positions in $\mathrm{X}, \mathrm{Y}$, and $\mathrm{Z}$ directions is plotted in Fig. 12(b). The mean and the standard deviation of the 2-norm of this difference are $1.4 \mu \mathrm{m}$ and $0.7 \mu \mathrm{m}$, respectively.

In this test, actual three-dimensional positioning accuracy at each command position was not measured. However, considering from linear positioning error, straightness error, and squareness error of each linear axis over the entire stroke, pre-calibrated by the machine tool's builder and also by us, three-dimensional positioning error within the test volume, $1 \times 1 \times 1 \mathrm{~mm}$, is estimated at most $1 \mu \mathrm{m}$ at each command position. 


\section{Case studies}

5.1 Case study 1: Calibration of an error map of a rotary axis by static measurement

(1) Test objective and procedure

This subsection demonstrates an experimental application of the developed non-contact R-test device to static measurement and presents its comparison with a conventional contact-type R-test device.

The experiment is conducted on a commercial small-size vertical-type five-axis machining center of the configuration shown in Fig. 13 (NMV1500DCG by Mori Seiki Co., Ltd.). The static R-test measurement cycle illustrated in Fig. 14 is conducted with the contact-type and non-contact R-test (see [8, 10] for further details of static R-test measurement cycle). The R-test measurement cycle is conducted with the following command B and C angular positions: $B_{i}^{*}=-75^{\circ},-50^{\circ}, \ldots, 75^{\circ}(\mathrm{i}=1, \ldots, 7)$, and $C_{j}^{*}=0^{\circ}, 30^{\circ}, \ldots, 330^{\circ}(\mathrm{j}=1, \ldots, 12)$. Total $7 \times 12=84$ points are measured.

The contact-type R-test used in this experiment is shown in Fig. 15 (see [10] for further details of this device). Table 5 shows specifications of the contact-type displacement sensor used in the contact-type R-test device.

(2) Test results

Figure 16 shows the graphical representation of the measured positions of the R-test sensors nest in the reference coordinate system when $B=0^{\circ}$ (see $[11,20]$ for more details on the graphical representation scheme of R-test results). From R-test measurement profiles, the sensors nest position in the reference coordinate system with respect to the spindle-side sphere can be observed at each $\mathrm{B}$ - and $\mathrm{C}$-angle. The measured profiles at $\mathrm{B}=50^{\circ}, 0^{\circ}$, and $-50^{\circ}$ are projected onto $\mathrm{XZ}$ plane, as plotted in Fig. 17. The errors are magnified 10,000 times (i.e. with an error scale of $10 \mu \mathrm{m} / 100 \mathrm{~mm}$ ). Note that the difference in the height (i.e. the distance from the table surface) of two profiles by contact-type and non-contact R-test 
devices in Figs. 16 to 17 is due to the difference in the Z-position of the sphere in both measurements.

From the test results, it can be observed that measured position profiles shown in Figs. 16 and 17 show a good agreement for contact-type and non-contact R-test devices within measurement uncertainty of each sensor (and the machine's positioning uncertainty).

5.2 Case study 2: Dynamic measurement with synchronous motion of a rotary axis and linear axes

(1) Test objective and procedure

A dynamic measurement is defined as a measurement that is conducted when the machine tool is driven with a velocity. To experimentally investigate the measurement performance of the developed non-contact R-test in a dynamic R-test measurement, the following experiment was conducted on the same five-axis machining center shown in Fig. 13.

The test procedure is shown as follows: 1) the C-axis is commanded to rotate with a constant angular velocity, 3,583 $\mathrm{deg} / \mathrm{min}$, at $\mathrm{B}=0^{\circ}$. The spindle-side sphere $(\mathrm{X}-$ and $\mathrm{Y}-$ axes) is commanded to follow the R-test sensors nest on the table (its feedrate is 5,000 $\mathrm{mm} / \mathrm{min}$ ). 2) The R-test continuously measures the relative displacement in the workpiece coordinate system. At the same time, the $\mathrm{C}$ angular position measured by a rotary encoder is logged.

Moreover, in this study, a set of Double Ball Bar (DBB) tests was conducted to compare with contact-type and non-contact R-test devices. The DBB measurement is widely applied to the circular test by two orthogonal linear axes $[21,22]$. The DBB tests presented in this section applied to a rotary axis have been studied by many researchers (e.g. [23]) and now included in ISO/DIS 10791-6:2012 [5]. In this study, the DBB device is set up to measure radial, tangential, and axial error motions of the C-axis with respect to synchronously 
controlled X- and Y-axes. The setups for the DBB measurement are illustrated in Fig. 18. Note that, due to the limitation in the experimental machine's $\mathrm{X}$ - and Y-axis strokes, the ball bar is inclined by about $66^{\circ}$ from the horizontal XY plane in the "tangential" setup, as illustrated in Fig. 18(b). The DBB device, DBB110 by Heidenhain, was used (reference length: $150 \mathrm{~mm}$ ). Figure 19 shows the DBB measurement setup in the axial direction (illustrated in Fig. 18(c)).

(2) Test results

For contact-type and non-contact R-tests, as illustrated in Fig. 20, the measured sphere displacement relative to the sensors nest is decomposed into the "radial", "tangential", and "axial" directions. In radial and tangential directions, the influence of the static center offset in X-and Y-direction is numerically eliminated, to focus on the dynamic errors only.

Figures 21, 22, and 23 shows measured displacement profiles in radial, tangential, and axial directions, respectively. In Figs. 21 (radial) and 22 (tangential), the measured sphere displacement is magnified 2,500 times and polar-plotted with respect to the C-axis feedback angular position. The start position of the C-rotation and the rotation direction are pointed out in the plots. The circle with "no displacement" represents the level where there is no displacement. The tangential error by the DBB shown in Fig. 22(c) is geometrically calculated by measured error profile in setups shown in Fig. 18(b) and (c). For tangential component shown in Fig. 22, negative error represents that the spindle-side sphere center is delayed relative to the R-test sensors nest on the table.

The following observations are made:

a. In all plots by the non-contact R-test, especially in the radial direction shown in Fig. 21(b), the "noise" (or high-frequency components of the measured profile) is larger (peak-to-peak $2 \mu \mathrm{m}$ approximately). It could be caused by the speckle noise in laser 
beam, as is also observed in profile scanning of a sphere shown in Fig. 7.

b. Except for the "noise", radial and axial trajectories (Figs. 21 and 23) by (a) contact-type, (b) non-contact R-test devices, and (c) the DBB show a good match.

c. However, in the tangential direction (Fig. 22), the measurement result with the contact-type R-test (Fig. 22(a)) shows a constant delay in the spindle-side sphere position relative to the R-test sensors nest (about $6 \mu \mathrm{m}$ ), which is not observed in the non-contact R-test and the DBB (Figs. 22(b) and (c)). This may show the measurement error by the contact-type R-test device due to the influence of the friction or the dynamics of sensors themselves.

d. To check the machine's repeatability, each of contact-type and non-contact R-tests and DBB measurements was repeated for three to five times. As an example, Fig. 24 shows all of three DBB measurements in the tangential direction (Fig. 22(c)). The measurement's repeatability is sufficiently high. No significant unsystematic error was observed in any of R-test and DBB measurements.

\section{Conclusion}

All the previous studies on the R-test in the literature used contact-type displacement sensors with a flat-ended probe. This paper presented the development of the non-contact R-test device using laser displacement sensors. A new algorithm was proposed to estimate the three-dimensional displacement of sphere center by using a non-contact R-test with laser displacement sensors, with considering the quasi-static measurement uncertainty caused by the sphere curvature.

A prototype non-contact R-test device was developed to experimentally verify the proposed algorithm to measure sphere center displacement. The measurement performance of the developed non-contact R-test device was investigated by comparing with the contact-type 
R-test. A potentially significant problem with the prototype non-contact R-test device is high-frequency noise of the amplitude about $2 \mu \mathrm{m}$, which is considered to be caused by the speckle noise in laser beam. From the profile measurement results of a sphere, when a laser displacement sensor of different principle is used (e.g. spectral interference type SI-F10 by Keyence), this noise is expected to be significantly reduced, although the measurable volume becomes smaller.

In the dynamic test to measure synchronization error of $\mathrm{C}$-axis rotation to the circular motion by $\mathrm{X}$ and Y-axes, except for this noise, measured error profiles by the non-contact R-test and the DBB match well within about $2 \mu \mathrm{m}$ in all of radial, tangential, and axial directions. On the other hand, in the tangential direction, the measurement result with the contact-type R-test shows a constant delay of about $6 \mu \mathrm{m}$, which is not observed in the non-contact R-test and the DBB. This may be caused by the friction between the sphere and sensor probes, or the dynamics of sensors themselves.

\section{Acknowledgement}

The machining center used in present experiments is loaned from the Machine Tool Technologies Research Foundation (MTTRF) through Equipment on Loan Award Program. This work is in part supported by a grant from Mitsutoyo Association for Science and Technology (MAST). It is also in part supported by the JSPS Grants-in-Aid for Scientific Research (\#23560122). 


\section{References}

[1] Ibaraki S, Knapp W, Indirect Measurement of Volumetric Accuracy for Three-axis and Five-axis Machine Tools: A Review, International Journal of Automation Technology 2012; 6(2); 110-124.

[2] Schwenke H, Knapp W, Haitjema H, Weckenmann A, Schmitt R, Delbressine F, Geometric Error Measurement and Compensation of Machines --An Update, CIRP Annals -- Manufacturing Technology 2008; 57(2); 560-575.

[3] Weikert S, R-Test, a New Device for Accuracy Measurements on Five Axis Machine Tools, Annals of the CIRP 2004; 53(1); 429-432.

[4] ISO 230-1:2012, Test code for machine tools -- Part 1: Geometric accuracy of machines operating under no-load or quasi-static conditions, 2012.

[5] ISO/DIS 10791-6:2012, Test Conditions for Machining Centers -- Part 6: Accuracy of Speeds and Interpolations, 2012.

[6] IBS Precision Engineering: http://www.ibspe.com.

[7] Fidia: http://www.fidia.it.

[8] Bringmann B, Knapp W, Model-based 'Chase-the-Ball' calibration of a 5-axis machining center, Annals of the CIRP 2006; 55(1); 531-534.

[9] ISO 230-7:2006, Test code for machine tools -- Part 7: Geometric accuracy of axes of rotation, 2006.

[10] Ibaraki S, Oyama C, Otsubo H, Construction of an error map of rotary axes on a five-axis machining center by static R-test, Int. J. of Machine Tools and Manufacture 2011; 51; 190-200.

[11] Hong C, Ibaraki S, Graphical presentation of error motions of rotary axes on a five-axis machine tool by static R-test with separating the influence of squareness errors of linear axes, Int. J. of Machine Tools and Manufacture 2012; 59; 24-33.

[12] Bringmann B, Maglie P, A method for direct evaluation of the dynamic 3D path accuracy of NC machine tools, CIRP Annals -- Manufacturing Technology 2009; 58(1); 343-346.

[13] Ota Y, Ibaraki S, Evaluation of dynamic errors of rotary axis in five-axis machining centers at the reversing point, Proc. of the JSME Mechanical Engineering Congress 2011, 2011 [in Japanese].

[14] Ihara Y, Hiramatsu Y, Design of motion accuracy measurement device for NC machine tools with three displacement sensors, International Journal of Automation Technology $2011 ; 5(6) ; 847-854$.

[15] Zargarbashi SHH, Mayer JRR, Single Setup Estimation of a Five-axis Machine Tool Eight Link Errors by Programmed End Point Constraint and On-the-fly Measurement with Capball Sensor, International Journal of Machine Tools and Manufacture 2009; 49(10); 759-766.

[16] Keyence: http://www.keyence.co.jp.

[17] Matlab, User's Guide: Optimization Toolbox for Use with Matlab, 2002.

[18] Seshagiri S, Khalil HK, Output Feedback Control of Nonlinear Systems Using RBF 
Neural Networks, IEEE Transaction on Neural Network 2000; 11(1); 69-79.

[19] Kimura Y, Matsubara A, Koike Y, Analysis of Measurement Errors of a Diffuse-Reflection-Type Laser Displacement Sensor for Profile Measurement, The 6th Int. Conf. on Leading Edge Manufacturing in 21st Century (LEM21), 2011.

[20] Hong C, Ibaraki S, Observation of thermal influence on error motions of rotary axes on a five-axis machine tool by static R-test, International Journal of Automation Technology 2012; 6(2); 196-204.

[21] Kakino Y, Ihara Y, Shinohara A, Accuracy Inspection of NC Machine Tools by Double Ball Bar Method, Hanser Publishers, 1993.

[22] ISO 230-4:2005, Test code for machine tools -- Part 4: Circular tests for numerically controlled machine tools, 2005.

[23] Tsutsumi M, Saito A, Identification and compensation of systematic deviations particular to 5-axis machining centers, Int'l J. of Machine Tools and Manufacture 2003; 43(8); 771-780. 


\section{Table and Figure Captions:}

\section{Table 1}

Main specifications of laser displacement sensors used in this study [16]

Table 2

Specifications of the reference sphere (from Moritex Co., Ltd)

Table 3

Summary of profile measurement of a ceramic sphere (diameter: $25.4 \mathrm{~mm}$ )

Table 4

Specifications of the reference sphere (from Kolb \& Baumann).

Table 5

Specifications of the linear displacement sensor (MT1281 from Heidenhain).

Fig. 1

Schematics of the R-test device.

Fig. 2

Influence of sphere center's offset on the measured displacement with contact-type displacement sensor and with non-contact type displacement sensor.

(a) By contact-type displacement sensor with flat-end probe

(b) By non-contact displacement sensor

Fig. 3

Measurement setup of conventional contact-type R-test.

Fig. 4

Measurement setup of non-contact R-test with three laser displacement sensors.

Fig. 5

Measurement uncertainty to the inclination of the target surface with specular reflection type laser displacement sensor (LK-G10).

Fig. 6

Schematic measuring principle of the laser displacement sensors [16].
(a) Specular reflection type (LK-G10)
(b) Diffuse reflection type (LK-H052)
(c) Spectral interference type (SI-F10)
(d) Confocal type (LT-9010MS)

Fig. 7

Profile measurement result of a sphere with specular reflection type laser displacement sensor (LK-G10).
(a) Measured profile
(b) Profile measurement error (projected onto XZ plane)

Fig. 8

Profile measurement result of a sphere with diffuse reflection type laser displacement sensor (LK-H052). 
(a) Measured profile

(b) Profile measurement error (projected onto XZ plane)

Fig. 9

Profile measurement result of a sphere with spectral interference type laser displacement sensor (SI-F10).

(a) Measured profile

(b) Profile measurement error (projected onto XZ plane)

Fig. 10

Profile measurement result of a sphere with confocal type laser displacement sensor (LT-9010MS).

(a) Measured profile

(b) Profile measurement error (projected onto YZ plane)

\section{Fig. 11}

Developed non-contact R-test device with three laser displacement sensors.

Fig. 12

Command and estimated sphere center positions within the volume $1 \times 1 \times 1 \mathrm{~mm}$.

(a) Command and estimated sphere center positions

(b) Difference in estimated and command sphere positions

Fig. 13

Configuration of the five-axis machine tool considered in this study.

Fig. 14

R-test measurement cycle $[8,10]$.

(a) At $B=0^{\circ}, \mathrm{C}$-axis is indexed at various angles. The spindle-side sphere is positioned by $\mathrm{XY}$ axes such that its relative displacement to the R-test sensors nest is nominally zero. Actual displacement is statically measured at each angle.

(b) Analogous measurement is done at various B angles.

Fig. 15

Contact-type R-test prototype [10,11,20].

Fig. 16

Measured sensor positions in the static R-test measurement cycle by contact-type and non-contact R-test devices (three-dimensional view).

Fig. 17

Measured sensor positions in the static R-test measurement cycle by contact-type and non-contact R-test devices, projected on XZ plane.
(a) At B $=50^{\circ}$
(b) At $\mathrm{B}=0^{\circ}$
(c) At $\mathrm{B}=-50^{\circ}$

\section{Fig.18}

Setups of a set of ball bar measurement to measure radial, tangential, and axial errors of the synchronous motion of $\mathrm{C}$-axis and $\mathrm{XY}$-axes. 

(a) Setup to measure the "radial" error
(b) Setup to measure the "tangential" error
(c) Setup to measure the "axial" error

\section{Fig.19}

Ball bar measurement setup in the axial direction of C-axis (Fig.18(c))

Fig. 20

Representation of the measured synchronous errors with synchronous motion of $\mathrm{C}$-axis and $\mathrm{X}$-, Y-axes.

\section{Fig. 21}

Measured synchronous errors in radial direction of $\mathrm{C}$-axis rotation.
(a) Contact-type R-test
(b) Non-contact R-test
(c) Ball bar

Fig. 22

Measured synchronous errors in tangential direction of $\mathrm{C}$-axis rotation.
(a) Contact-type R-test
(b) Non-contact R-test
(c) Ball bar

\section{Fig. 23}

Measured synchronous errors in axial direction of $\mathrm{C}$-axis rotation.
(a) Contact-type R-test
(b) Non-contact R-test
(c) Ball bar

\section{Fig. 24}

Repeated ball bar measurements (three times) of synchronous error of C- to XY-axes in tangential direction (feedrate: $5,000 \mathrm{~mm} / \mathrm{min}$ ). 


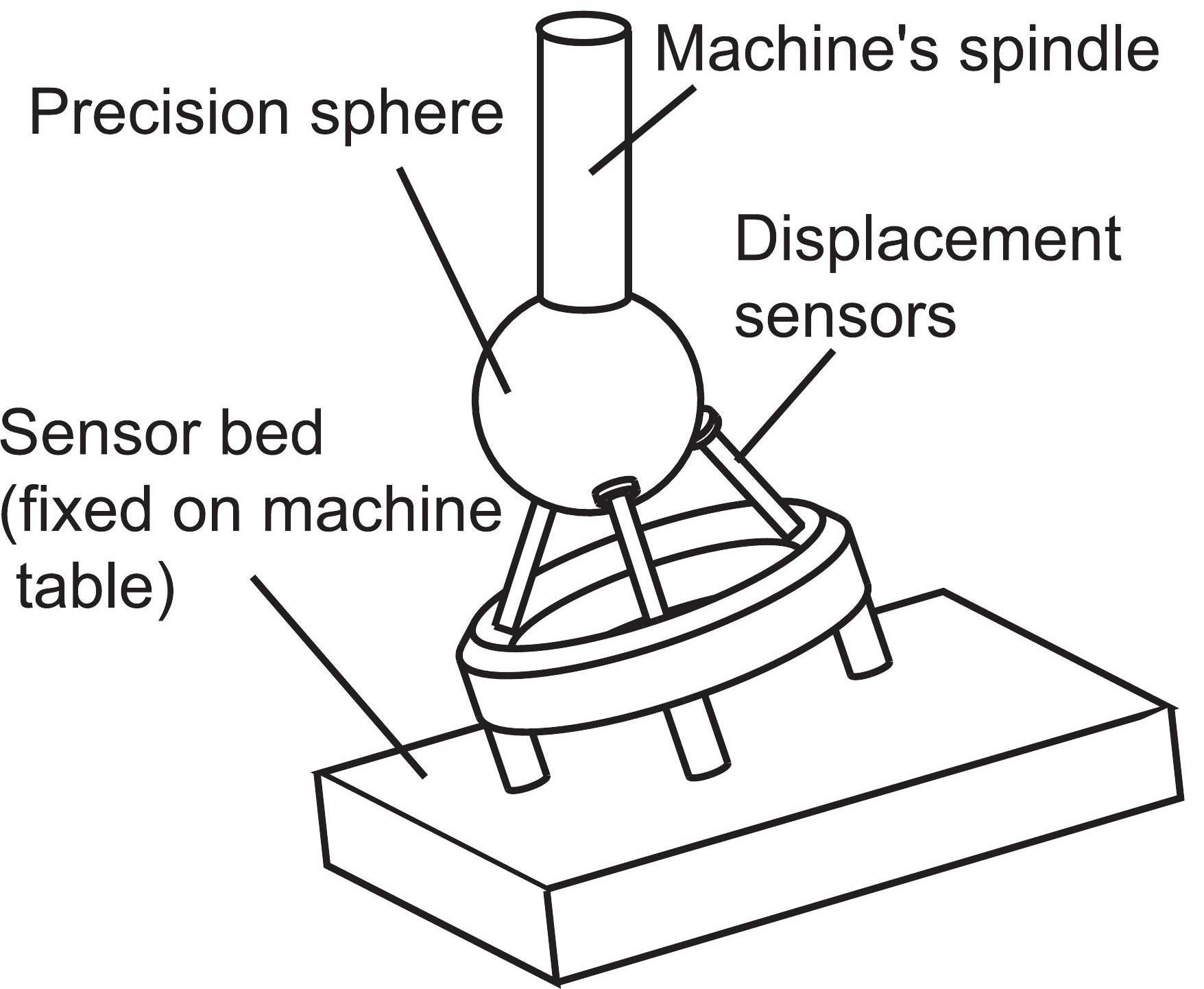


sphere positionl after offset

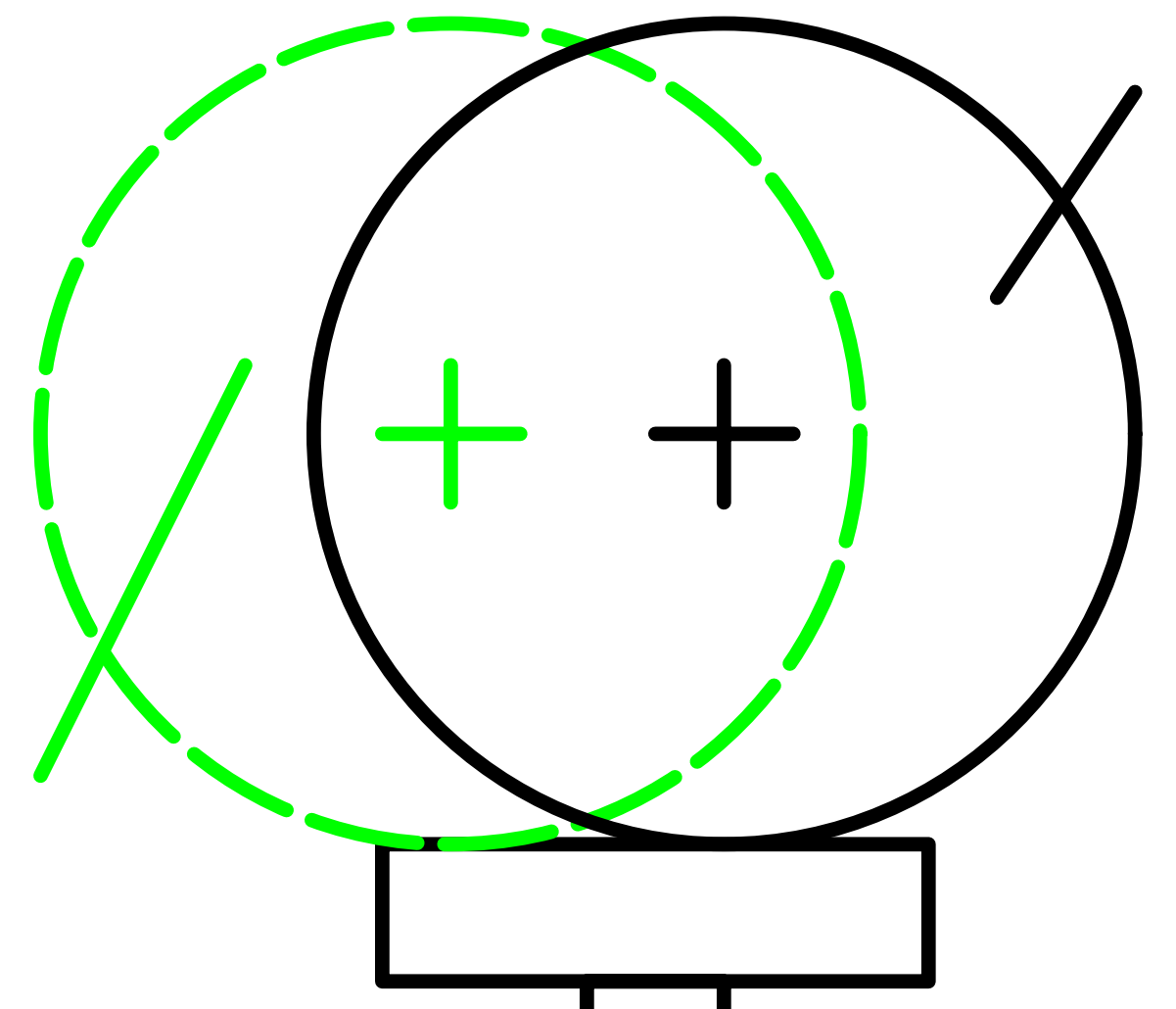

initial sphere position

\section{contact-type displacement sensor}




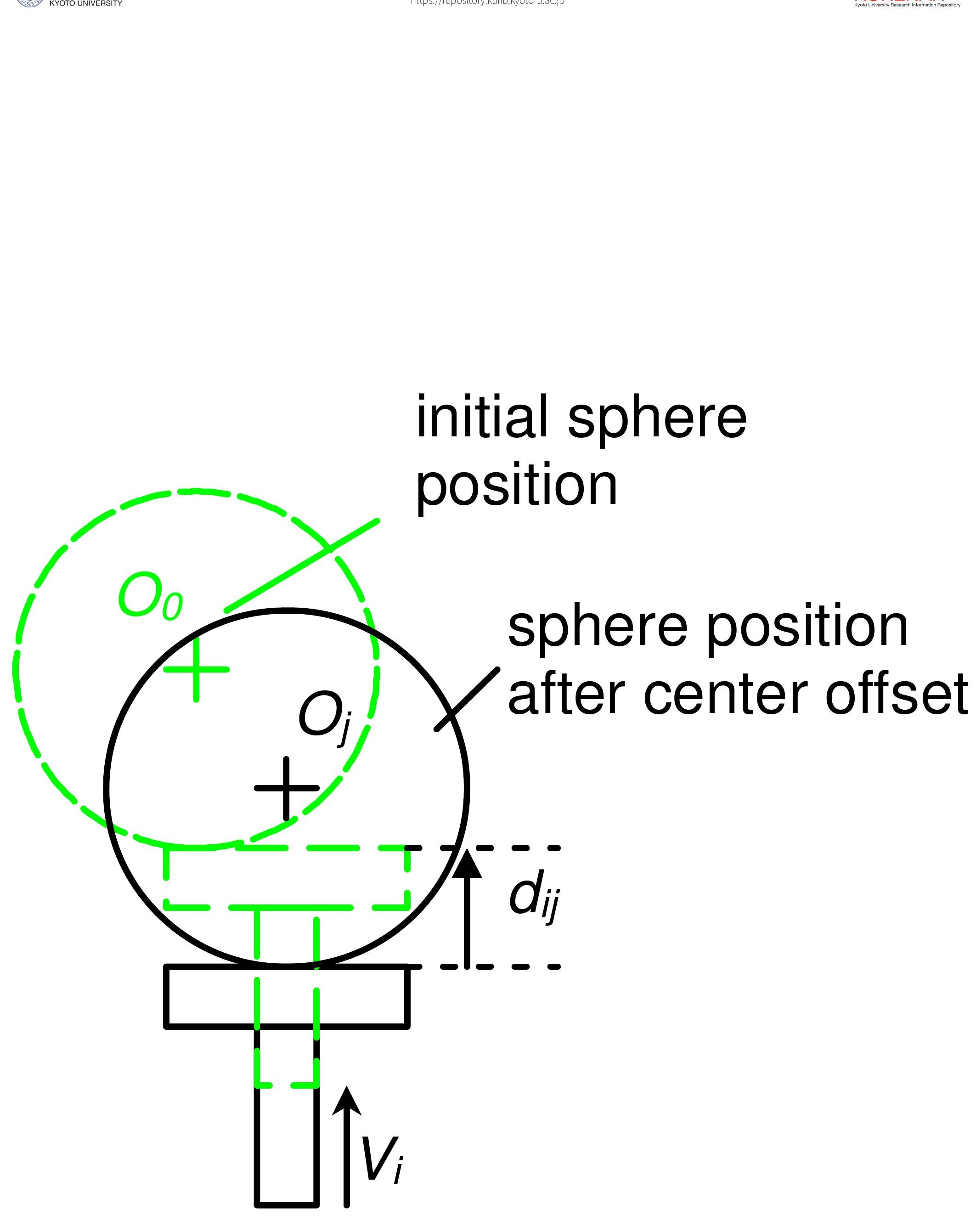

initial sphere position

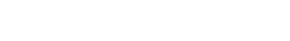

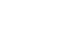

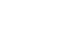
here 


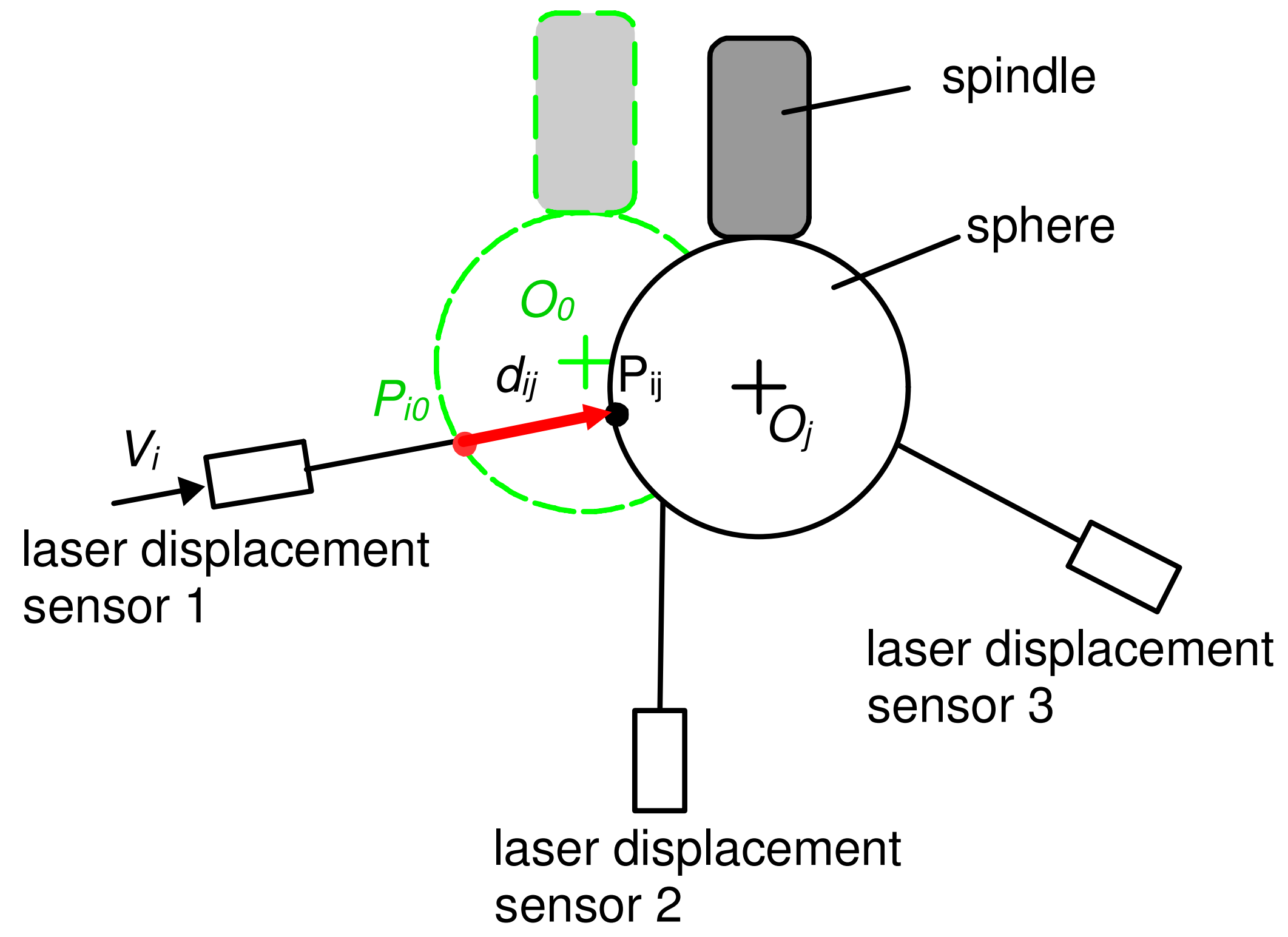




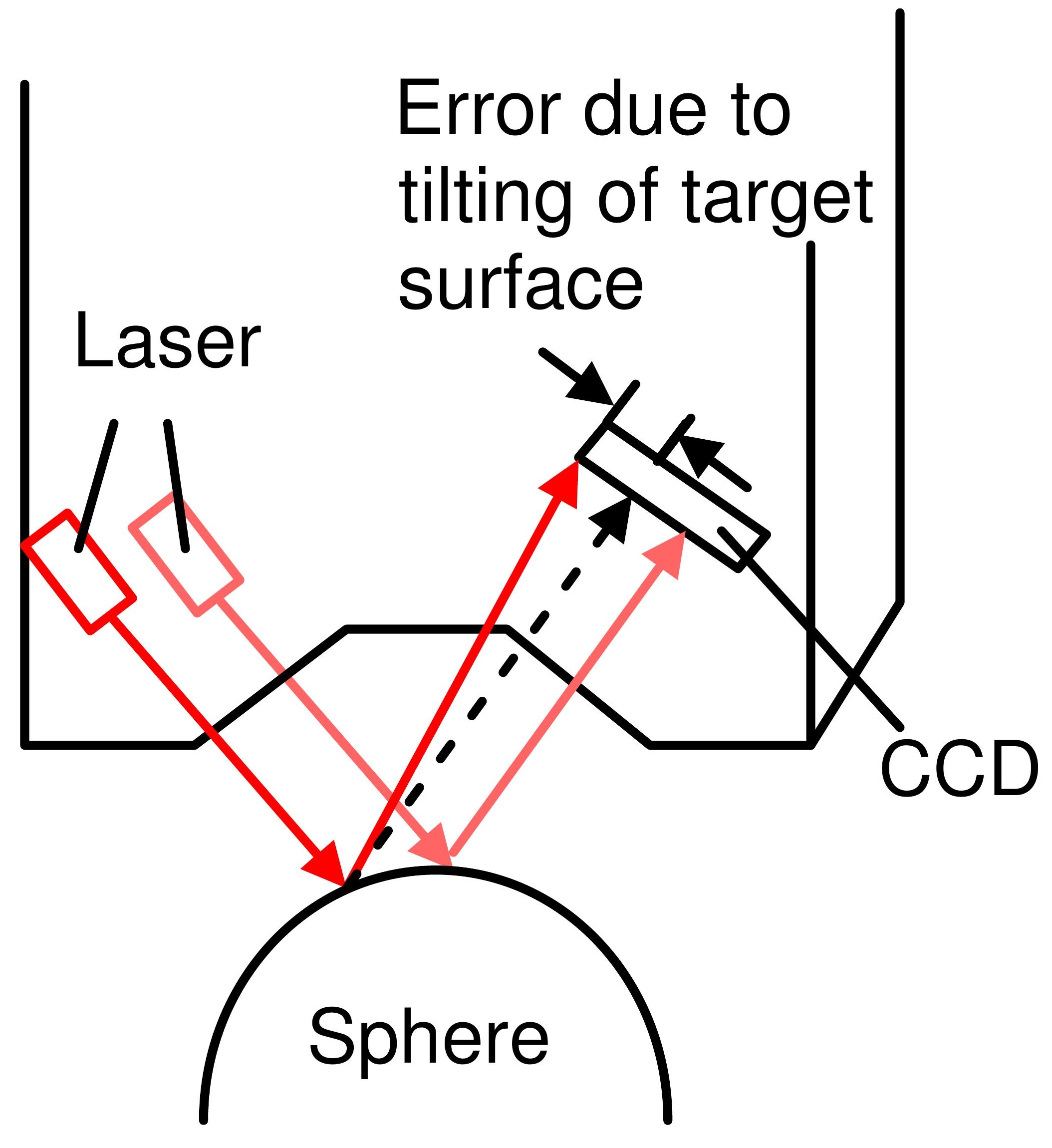




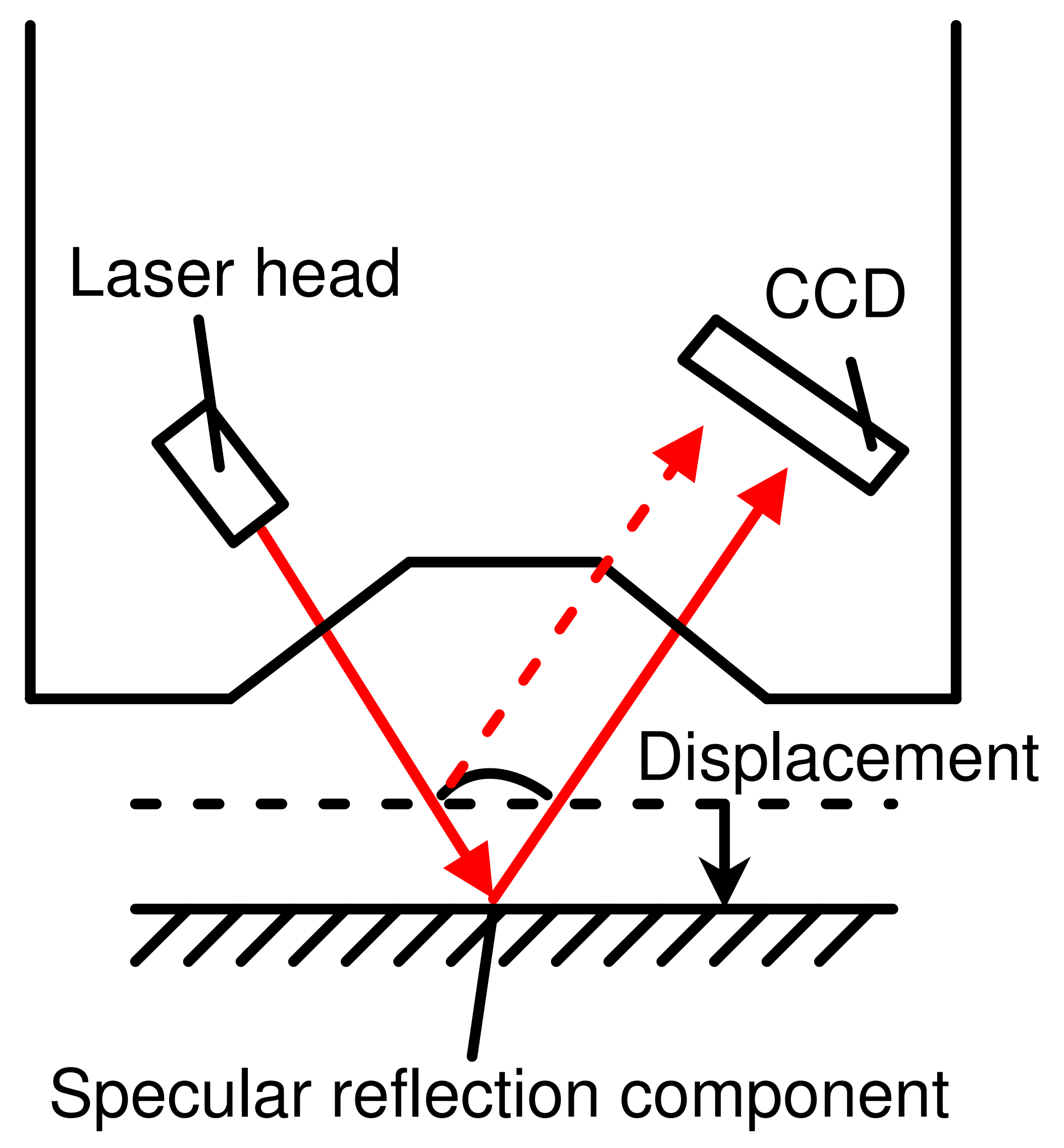




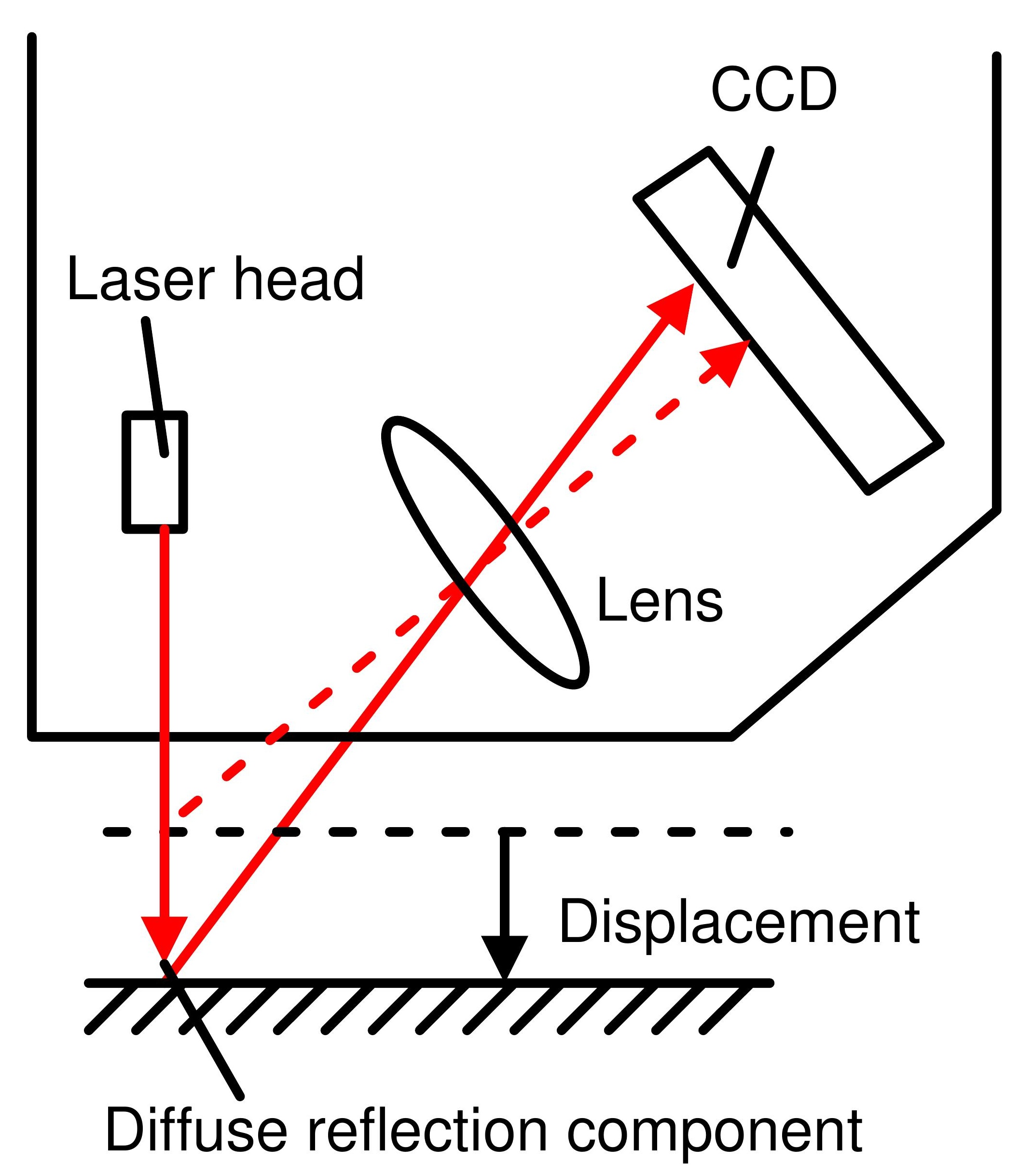


'Laser head (Multi-

wavelength)

1

Interference

CCD
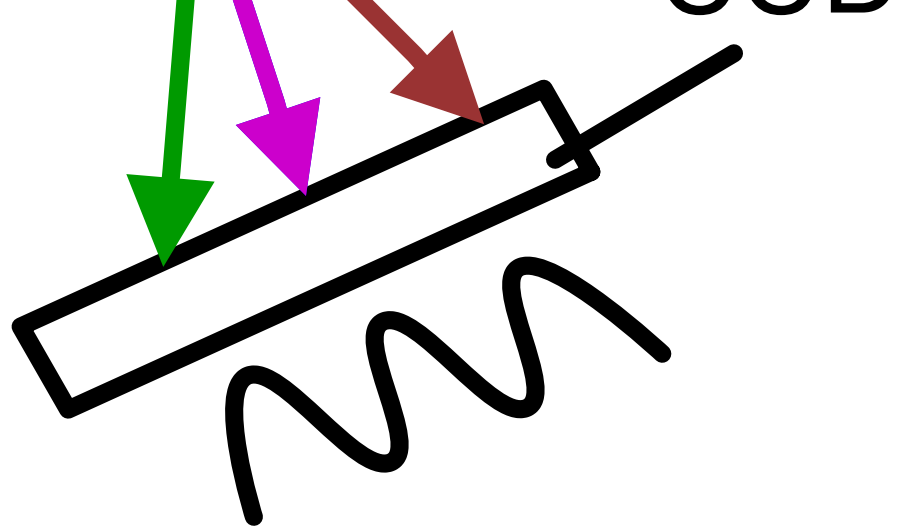

Displacement is analyzed from the spectrum.

\section{Reference}

- ..... [ . - .

$\downarrow$ Displacement 


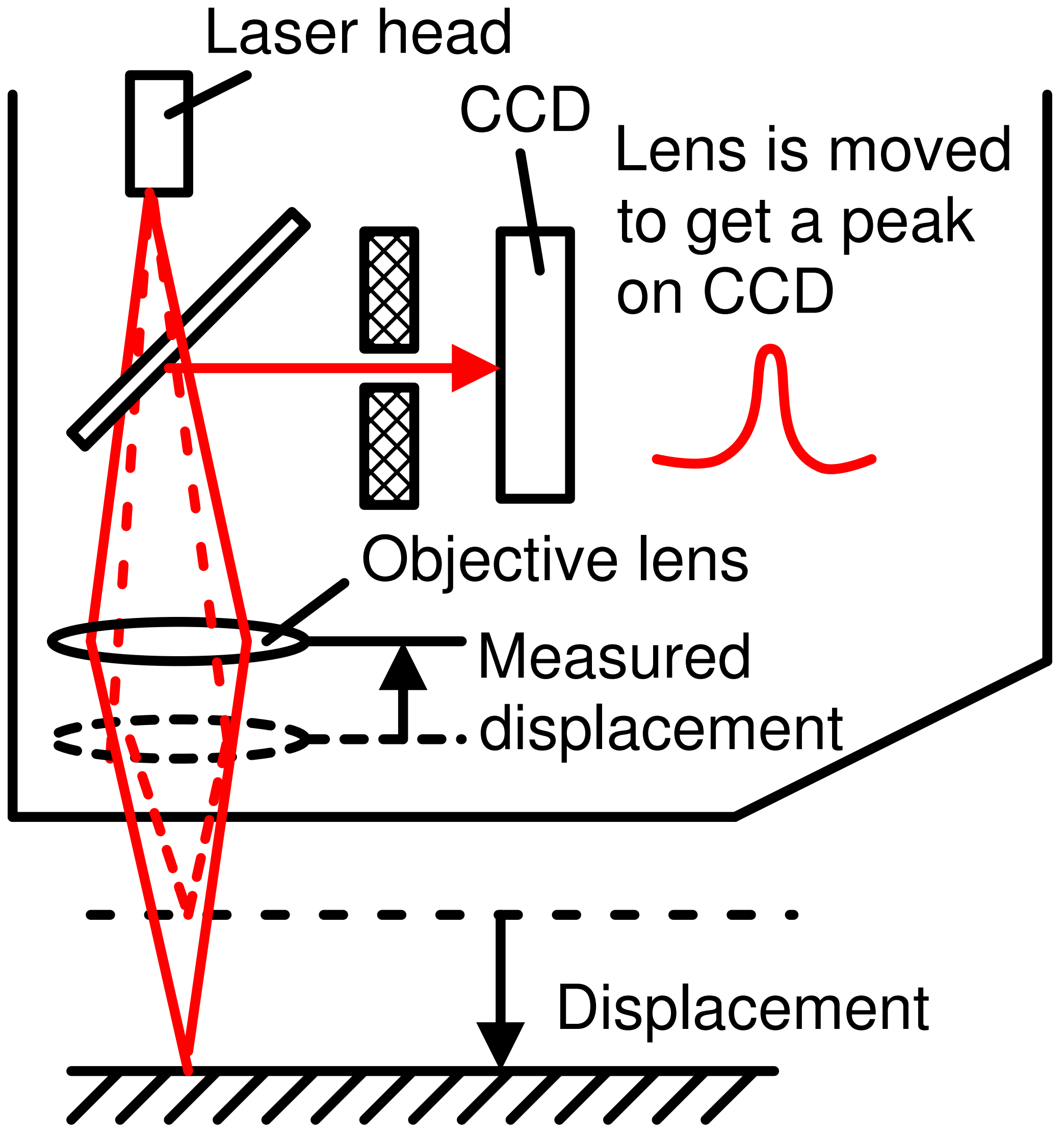




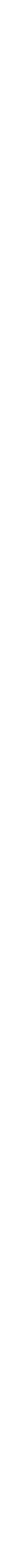




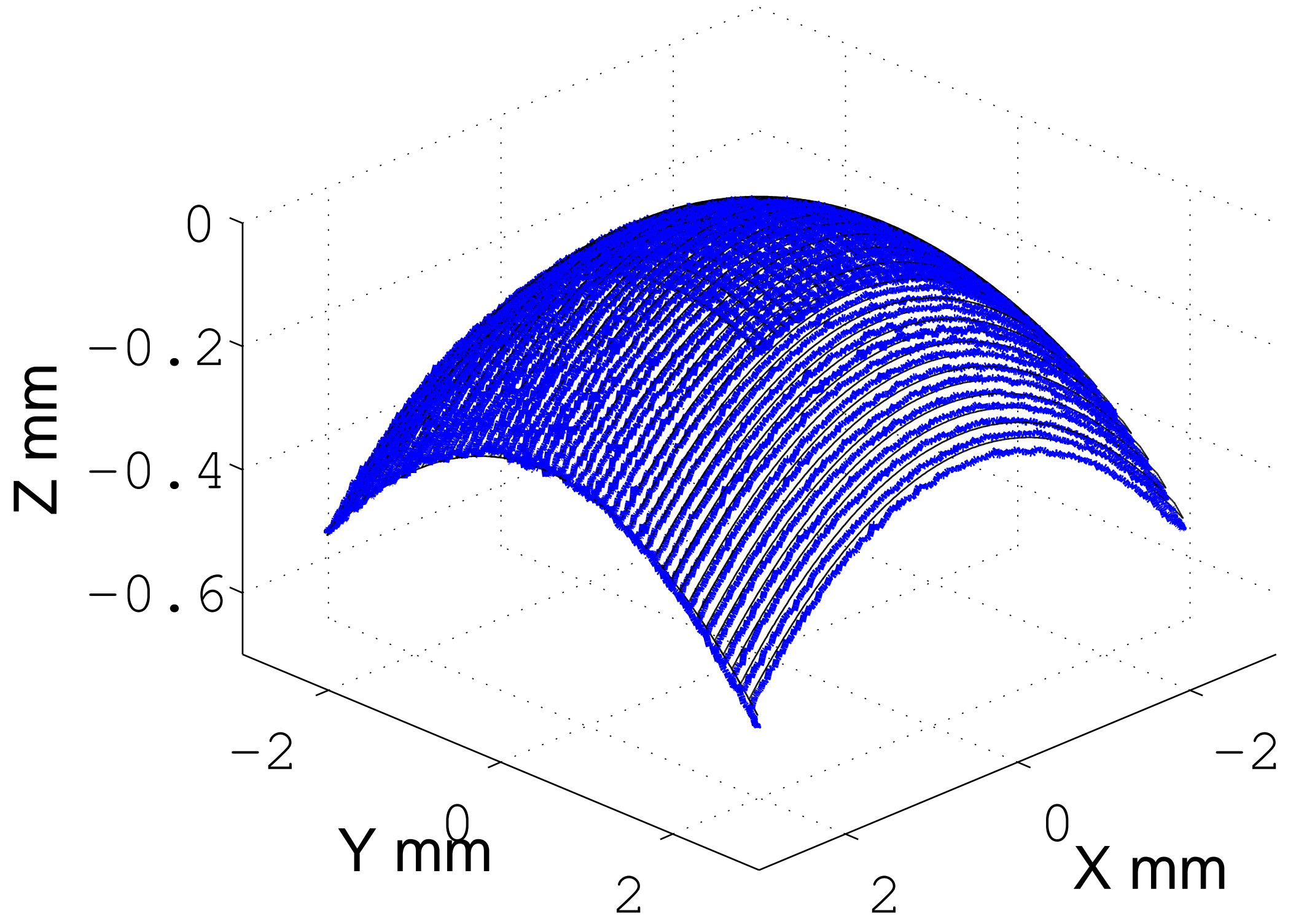




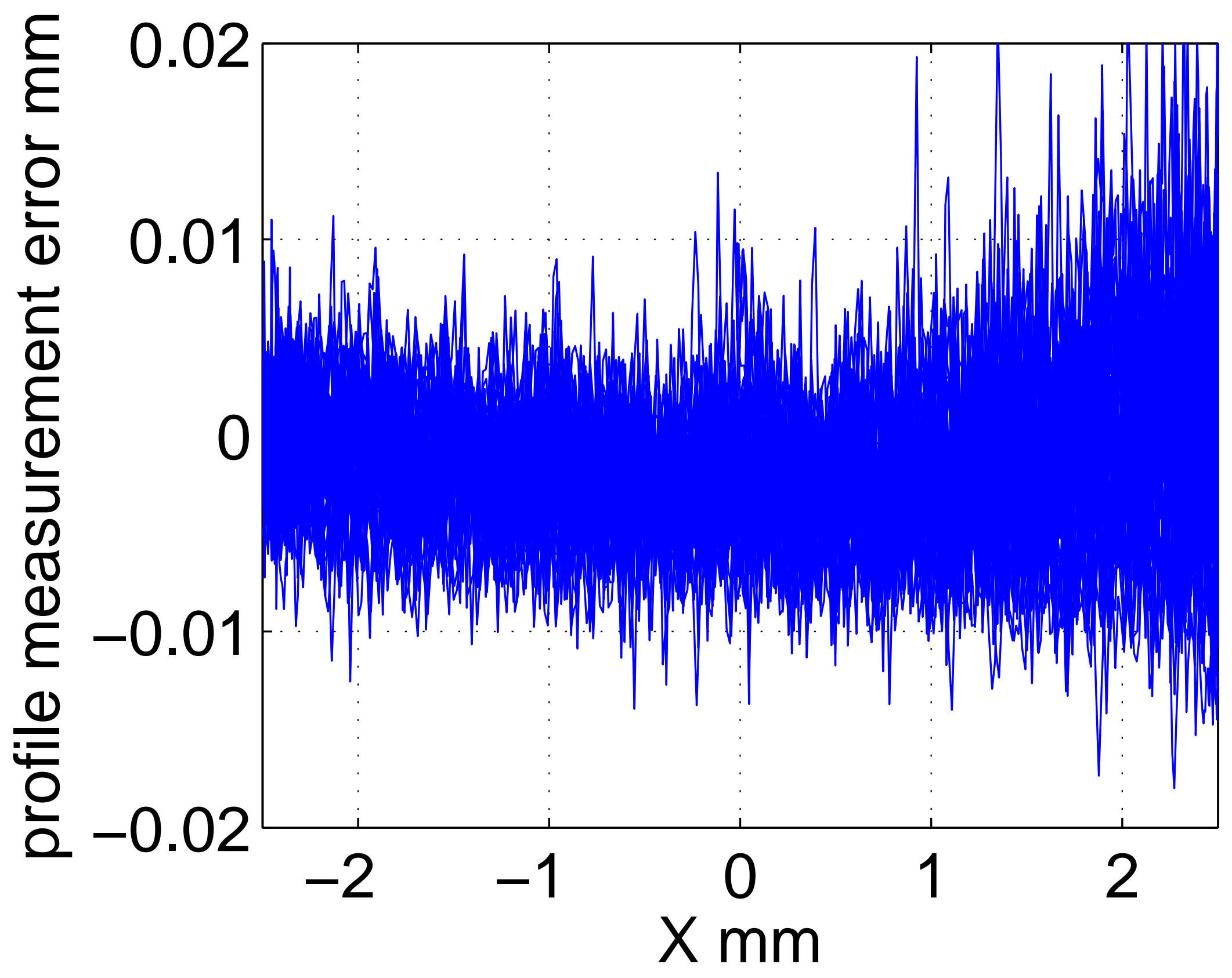




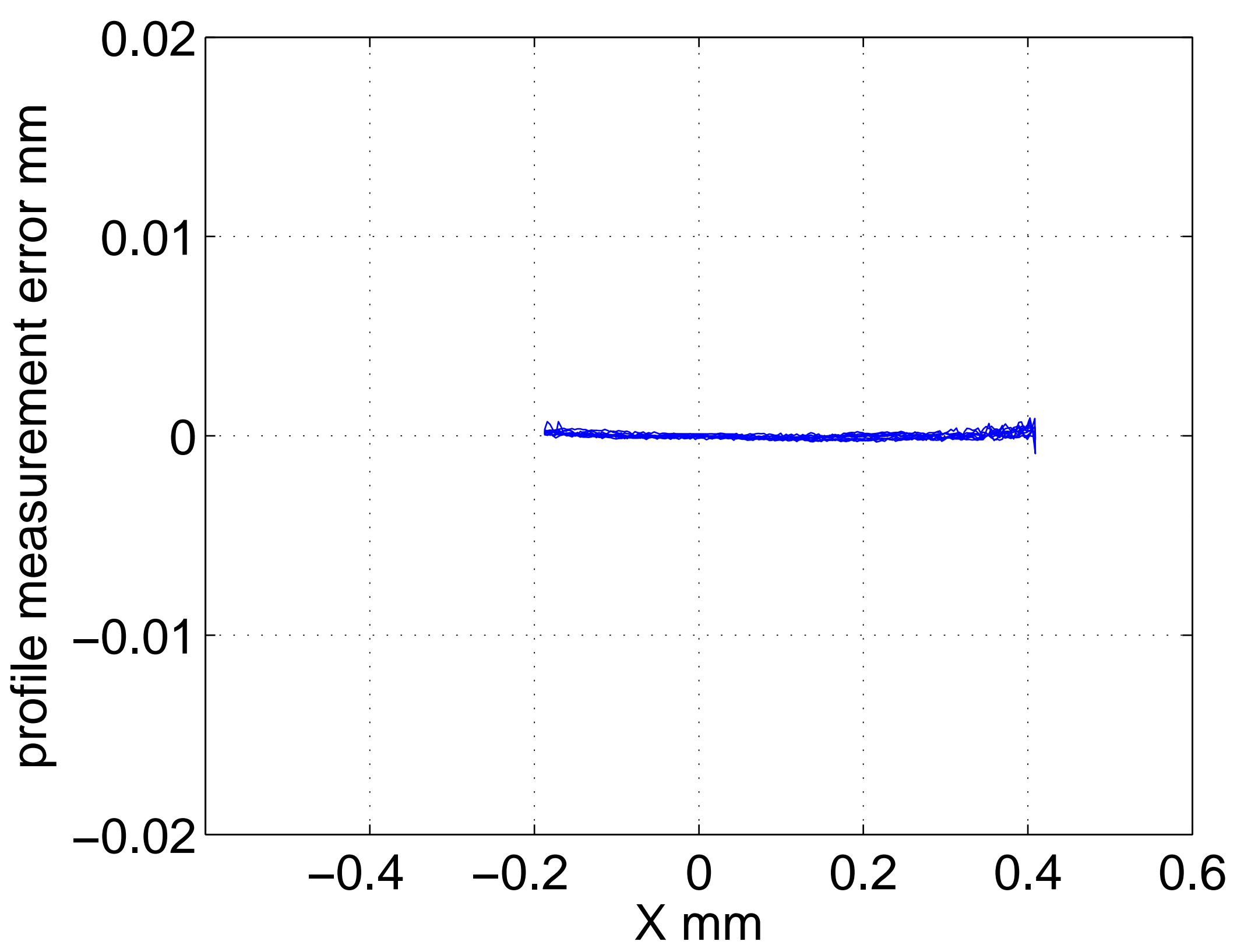




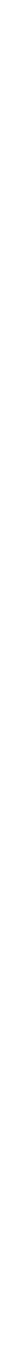




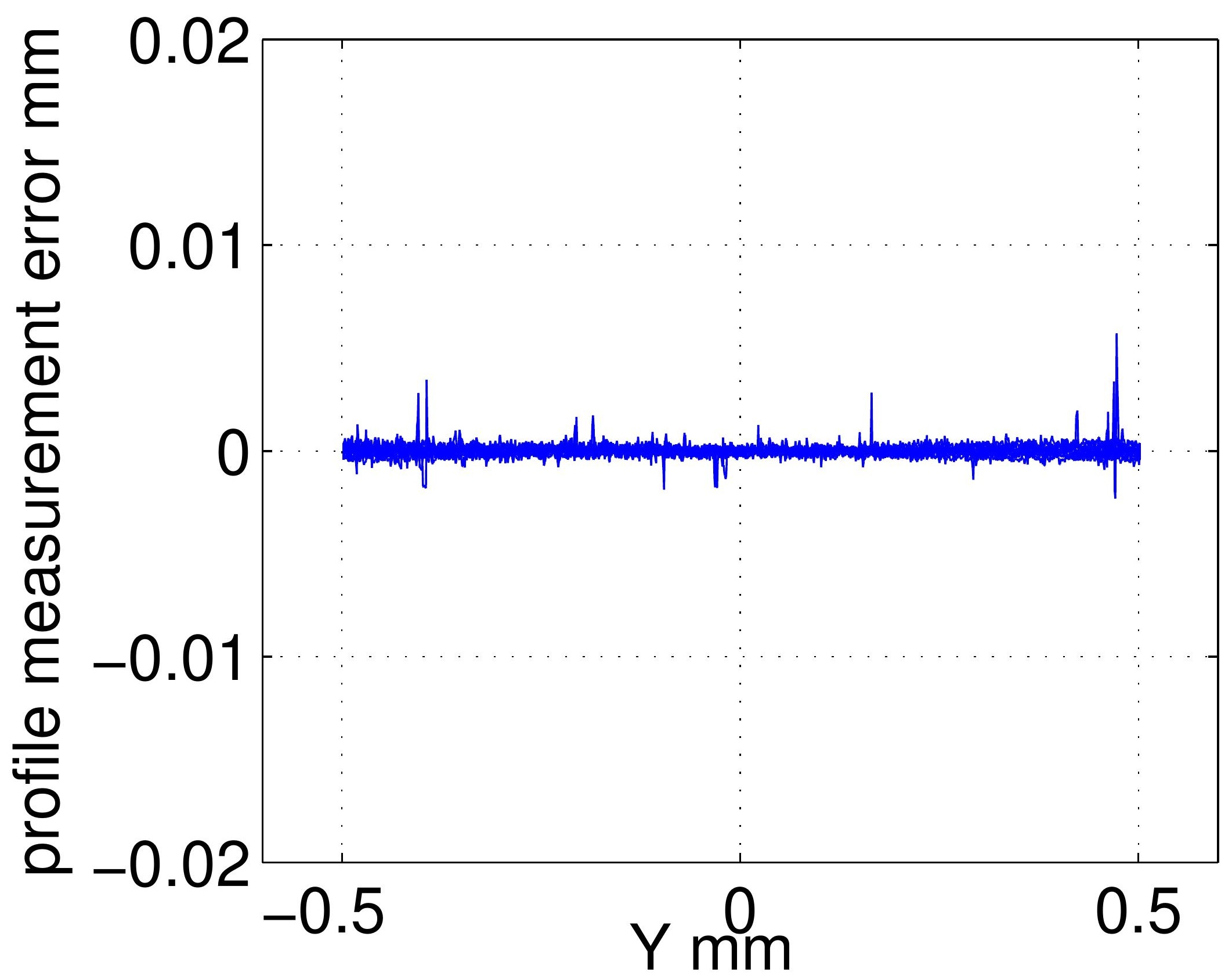


(

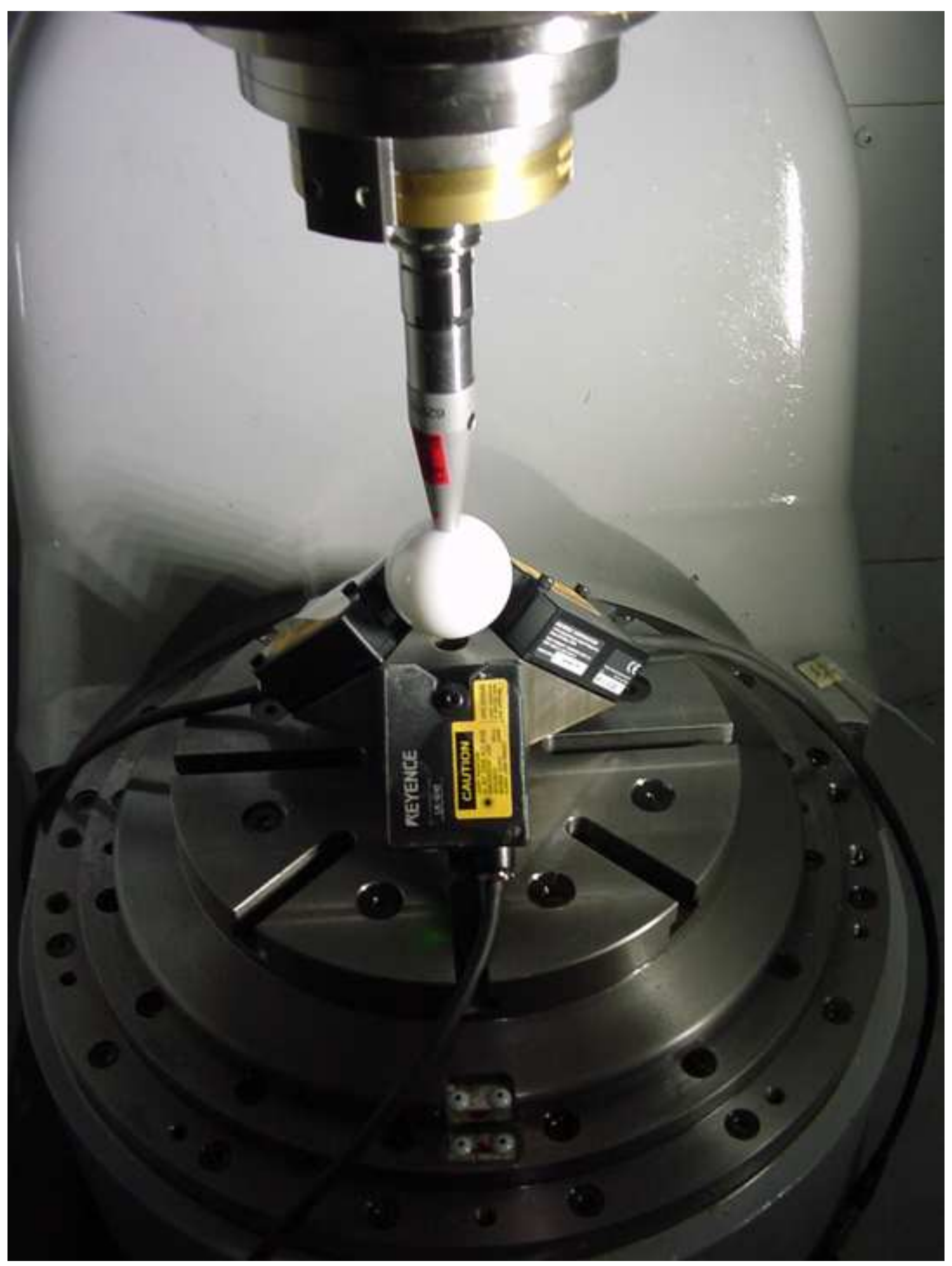




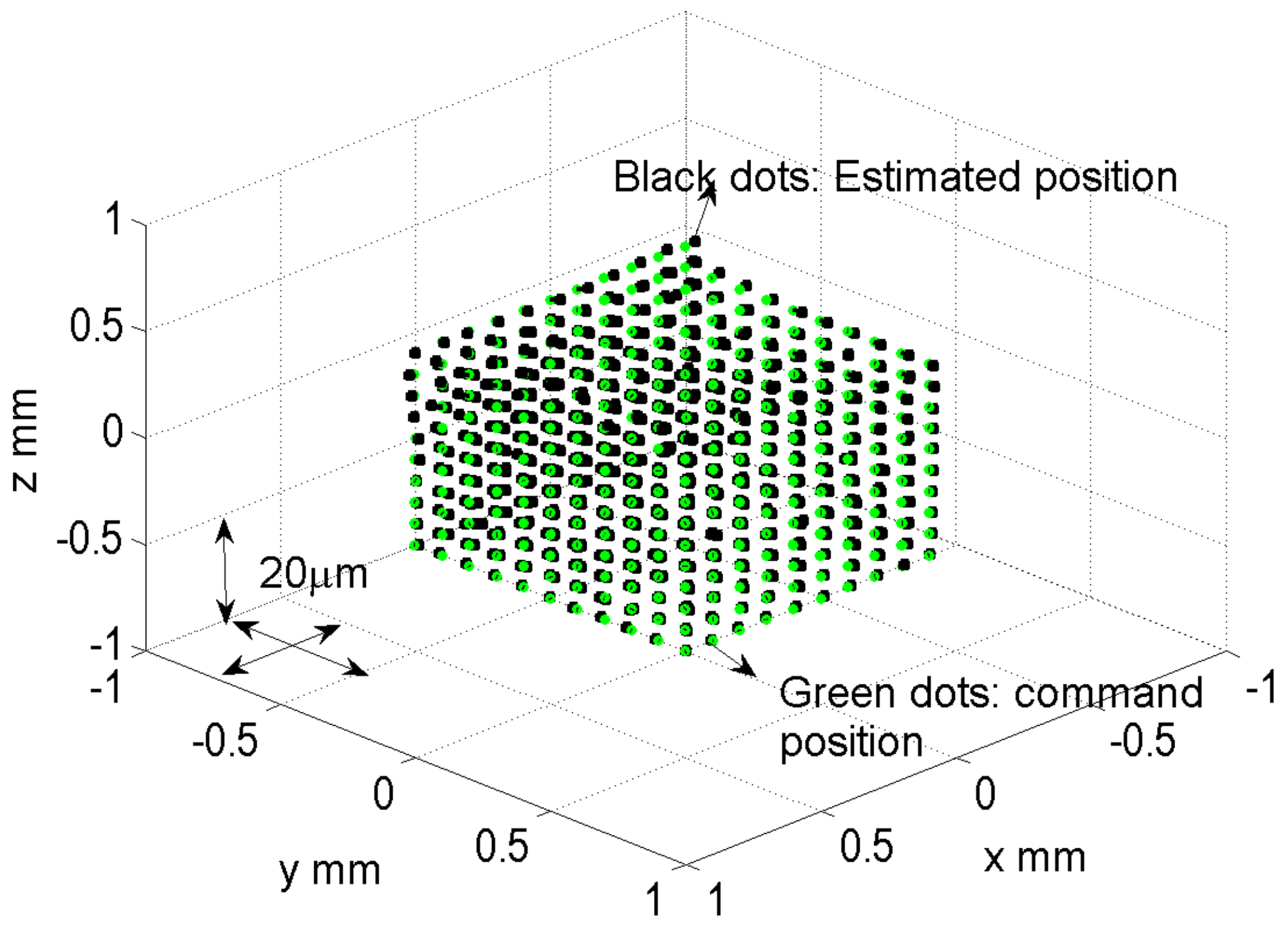




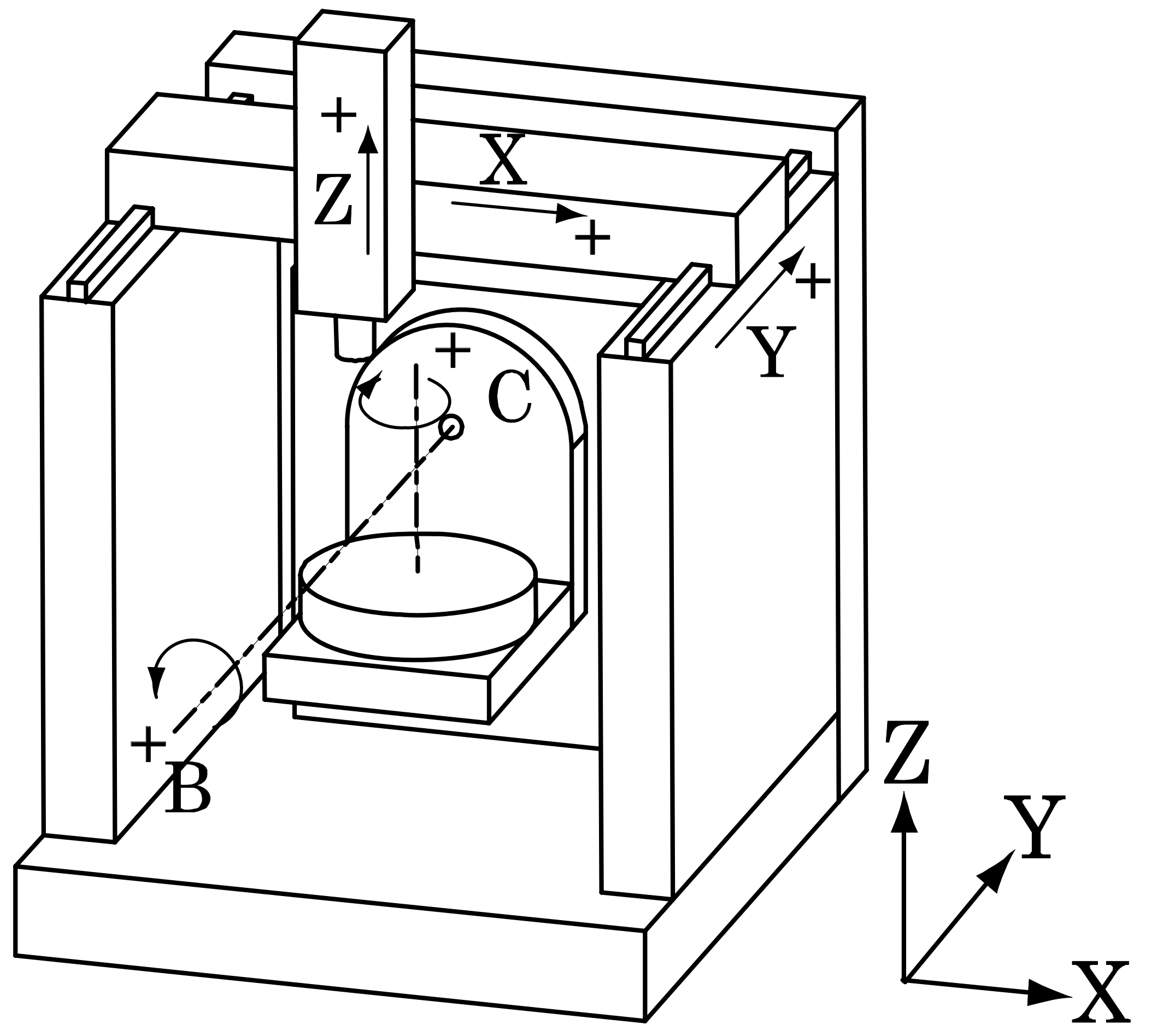




\section{$\mathrm{C}=90^{\circ}, 120^{\circ}, \ldots, 330^{\circ}$}

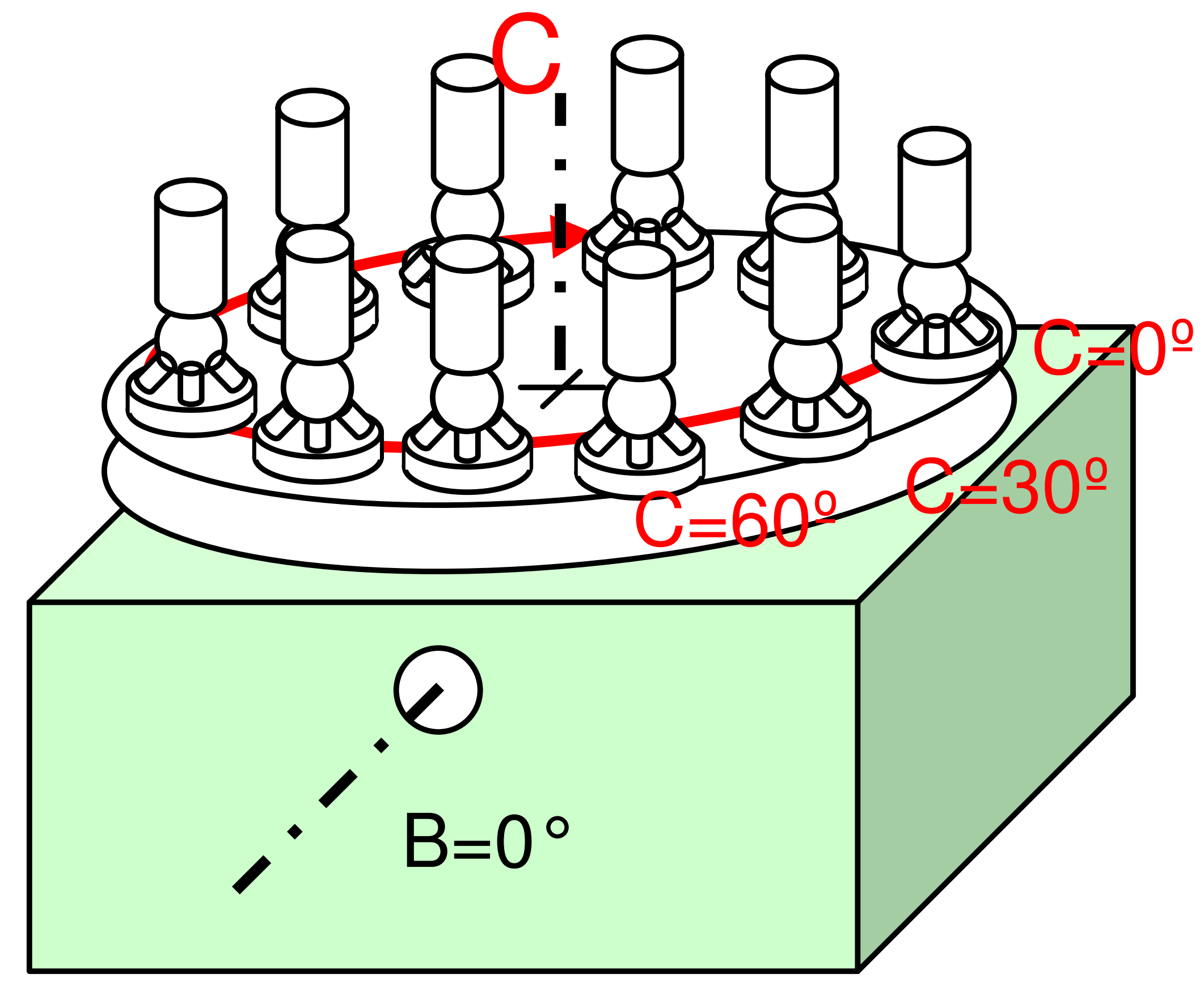




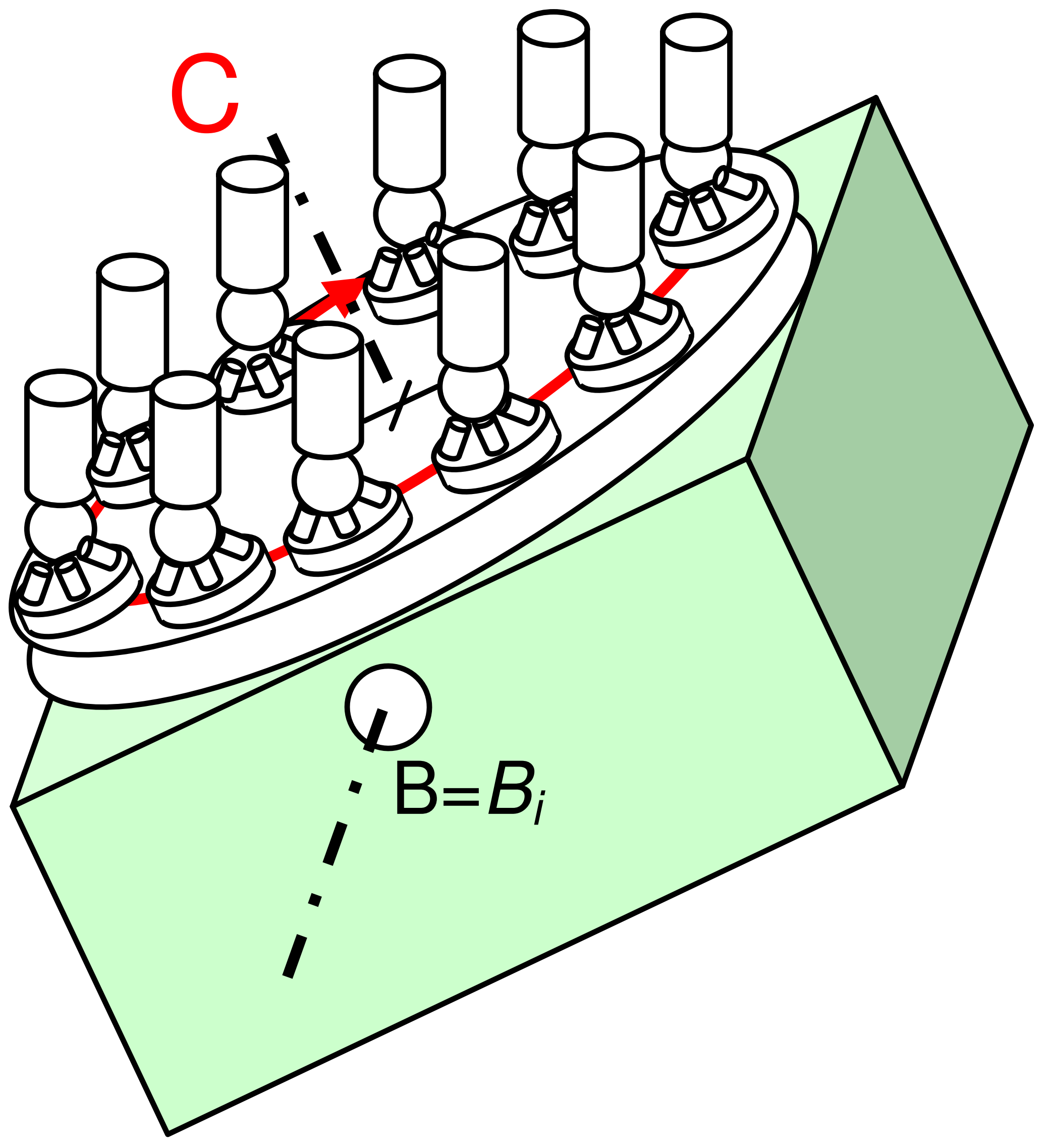




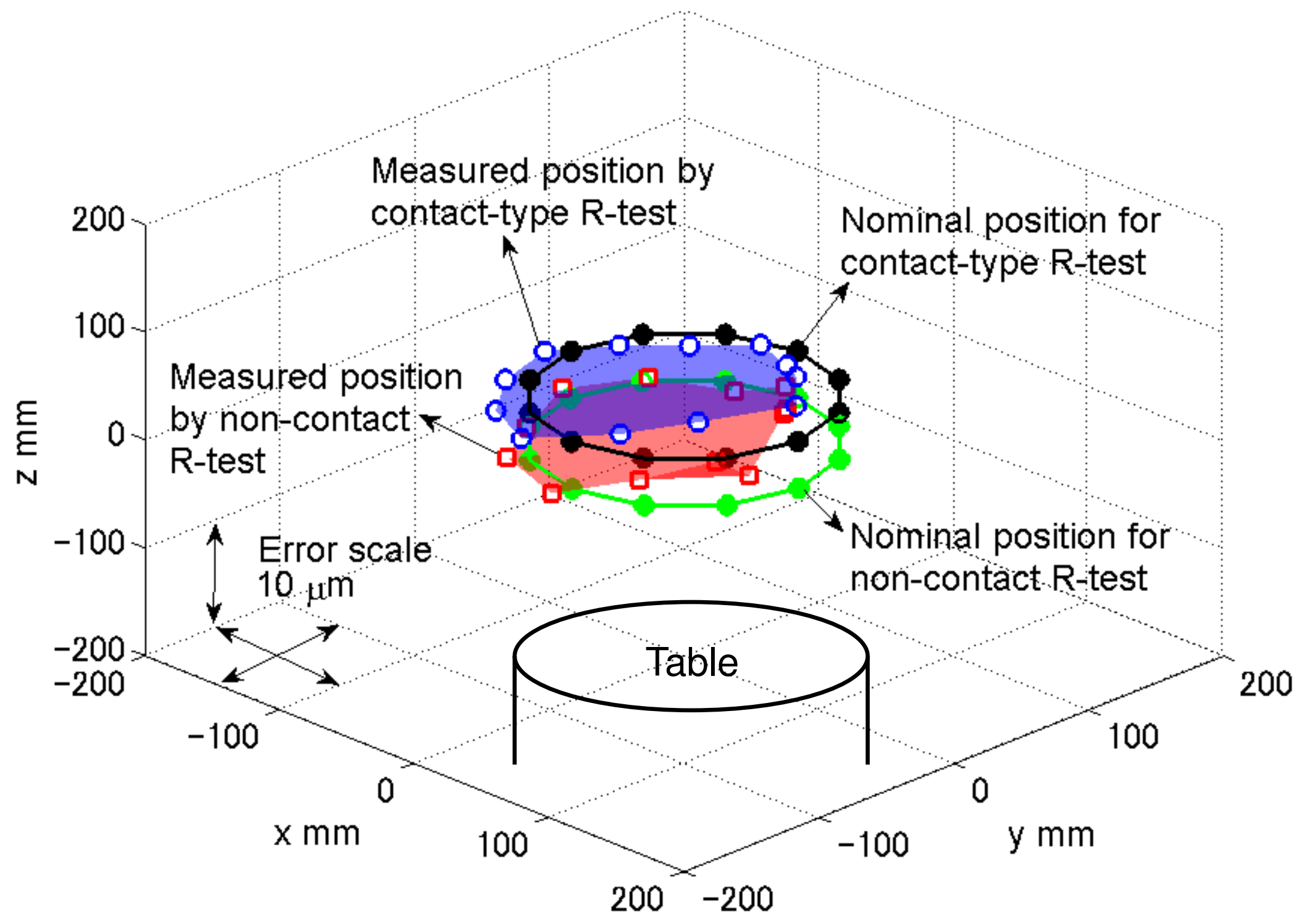




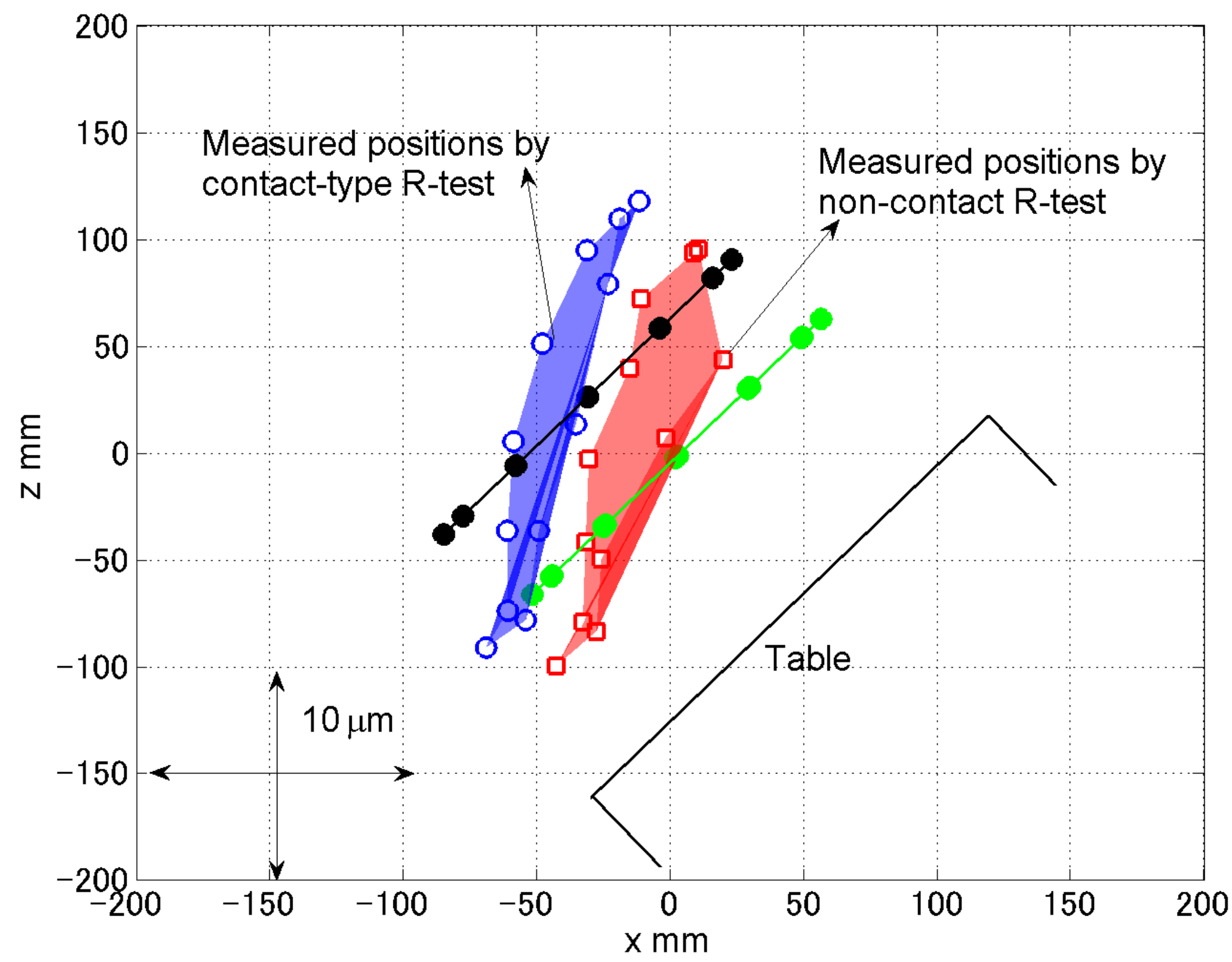




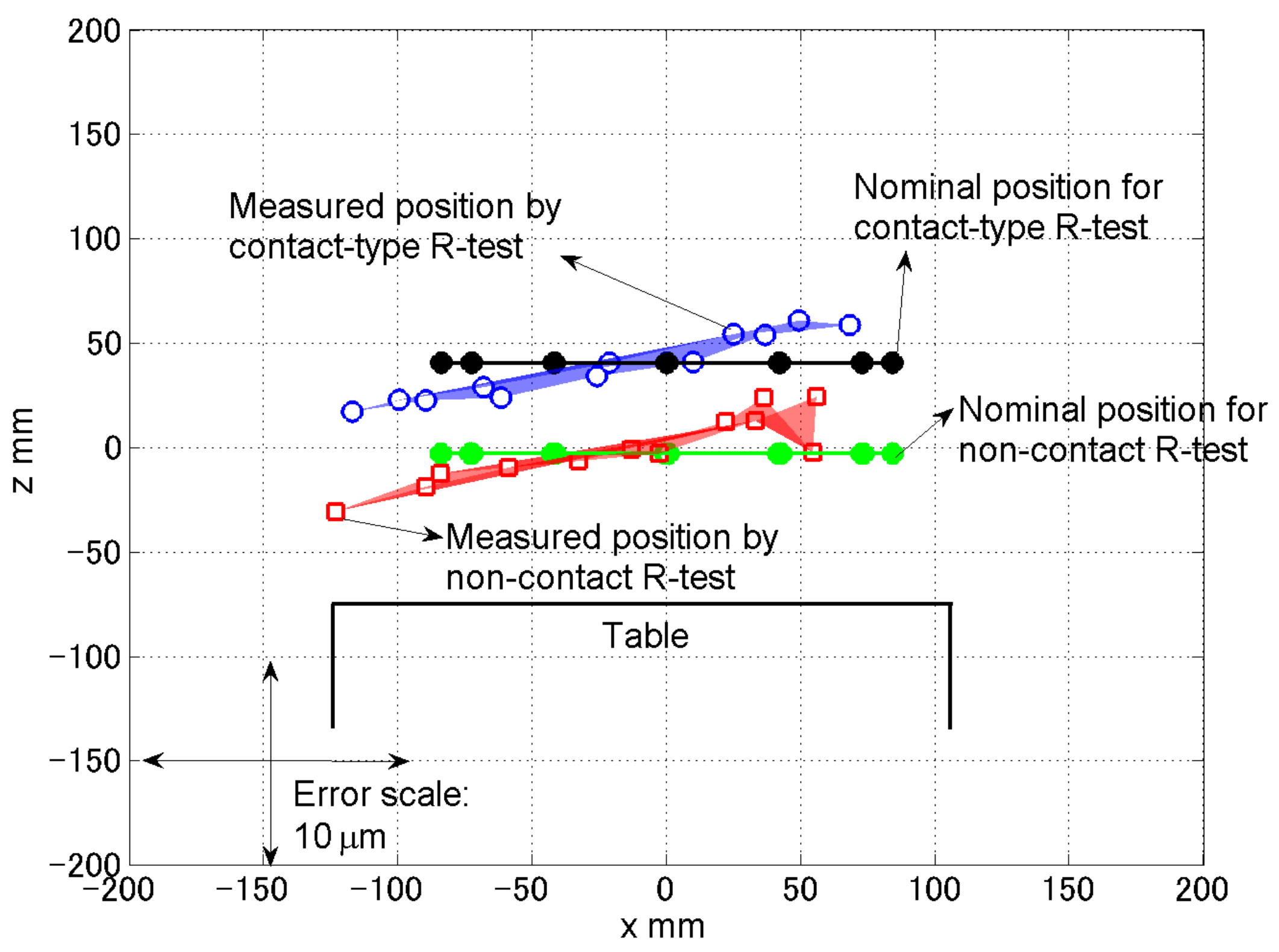




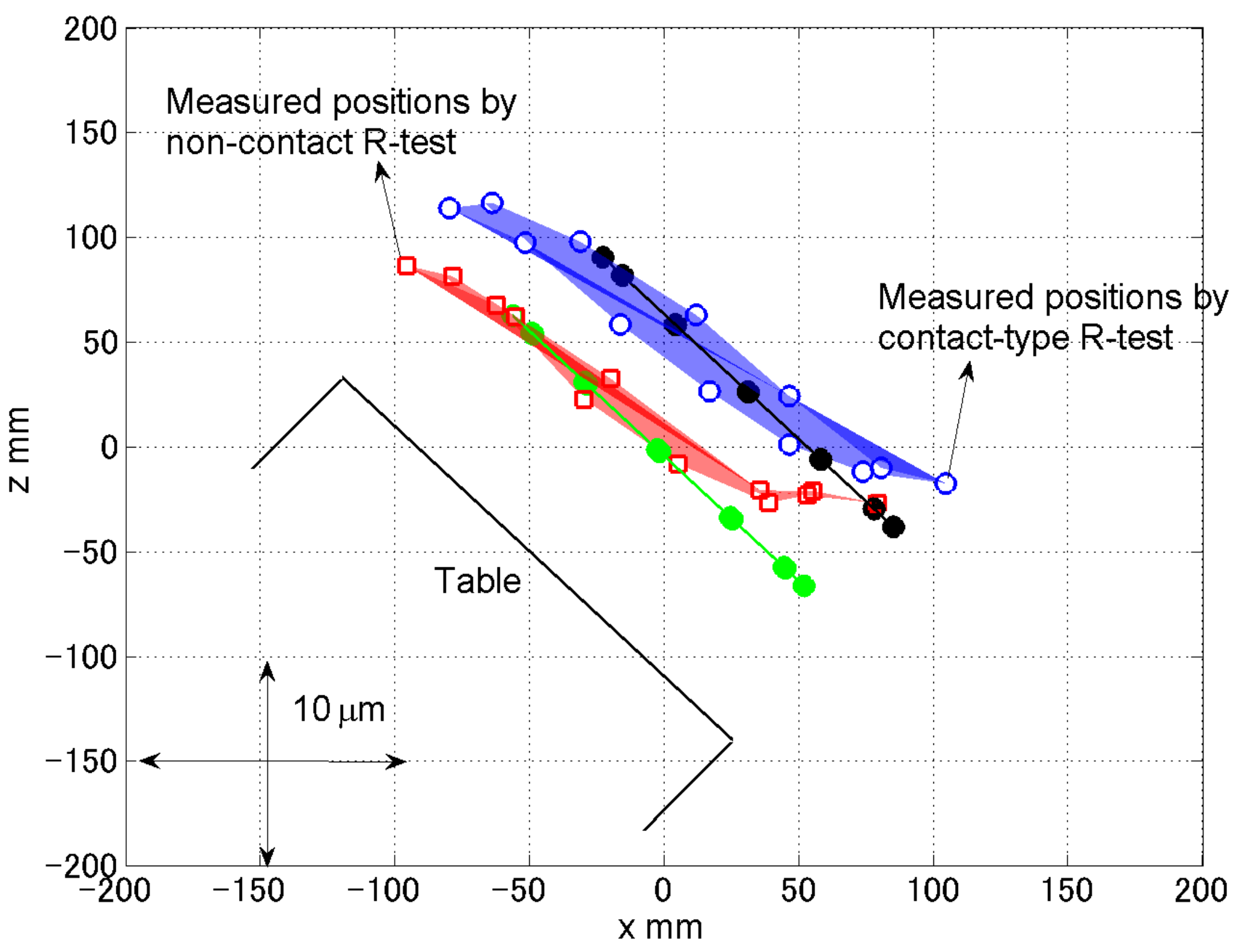




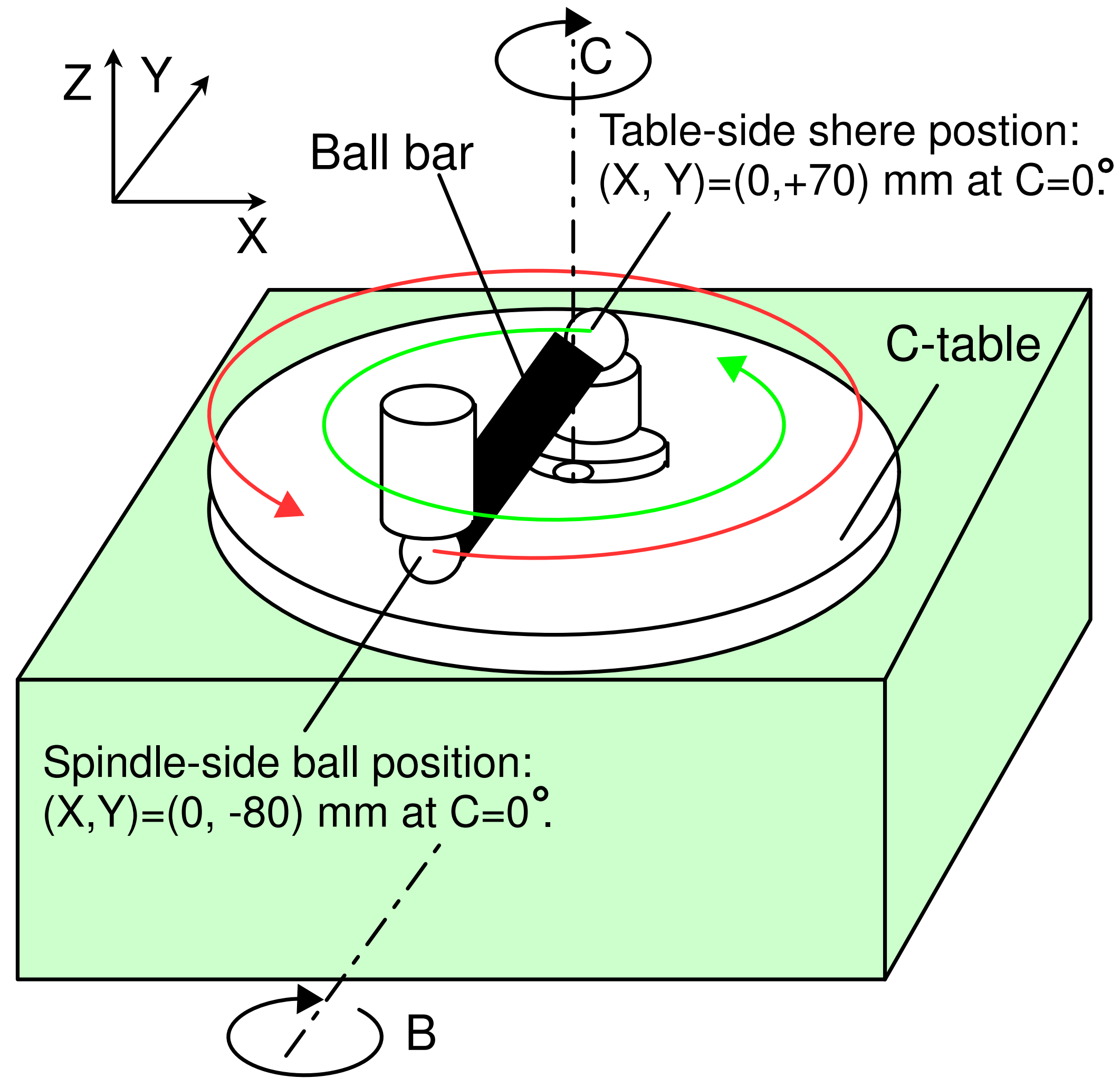




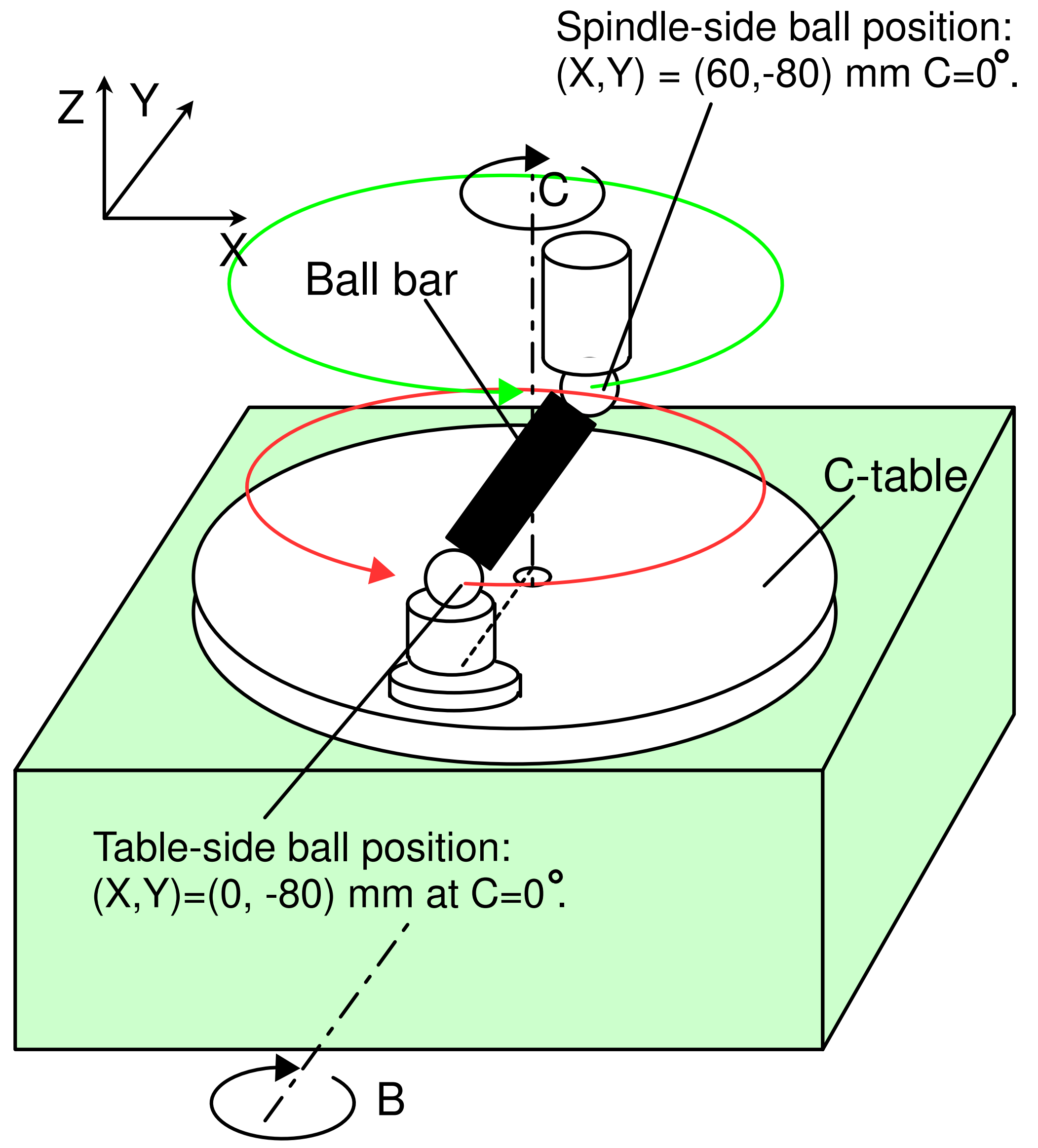




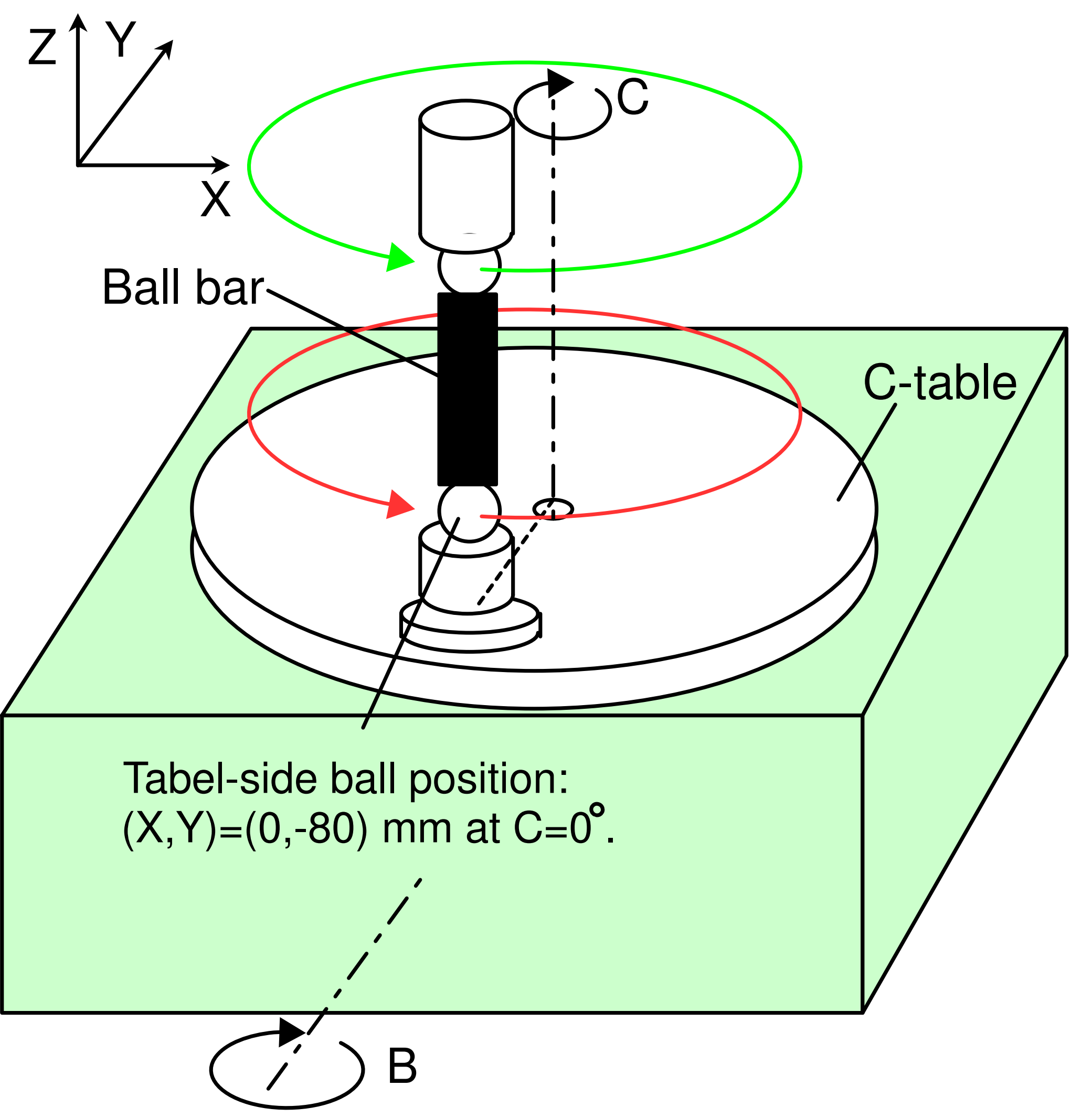


F: 都大学

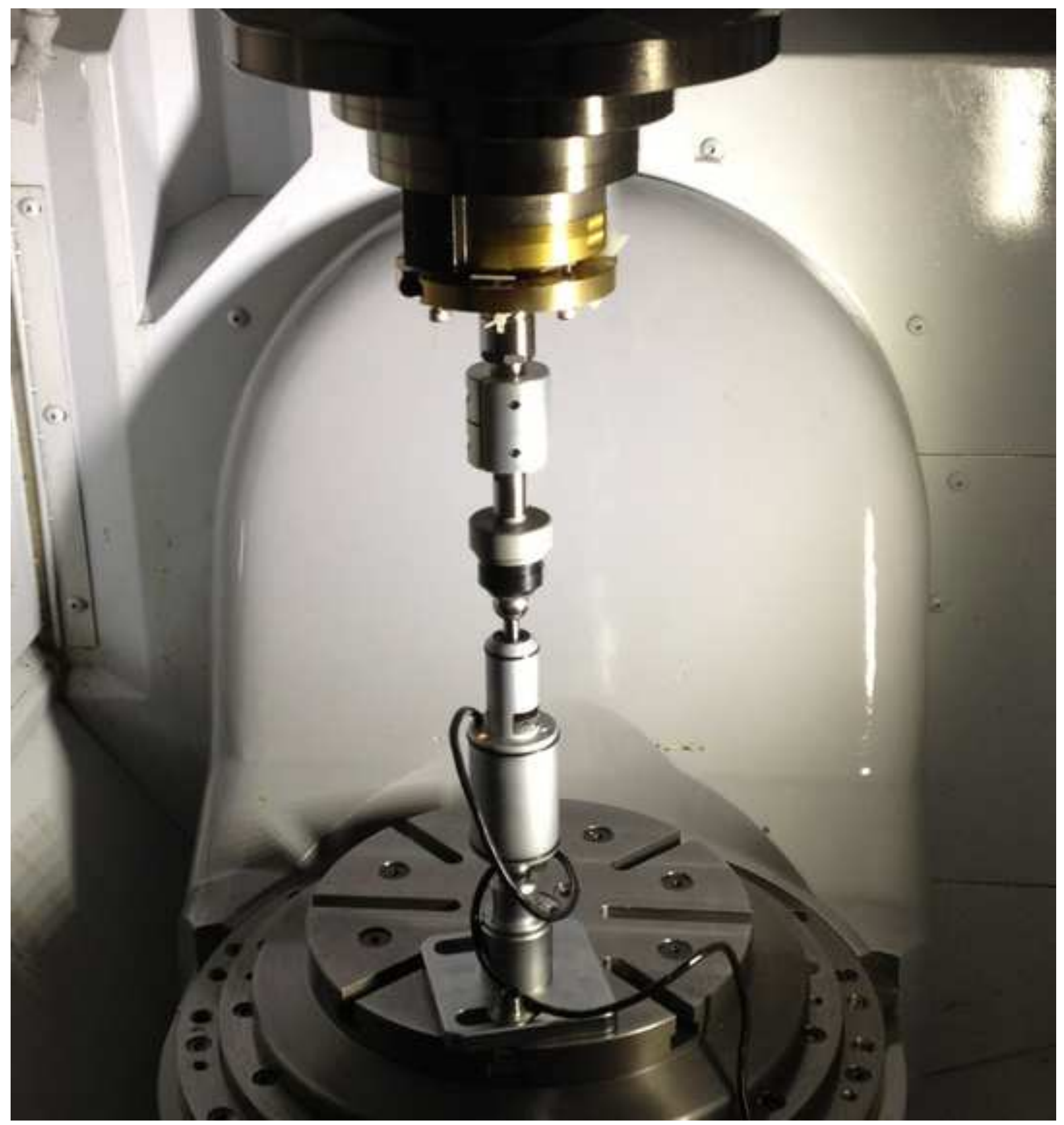




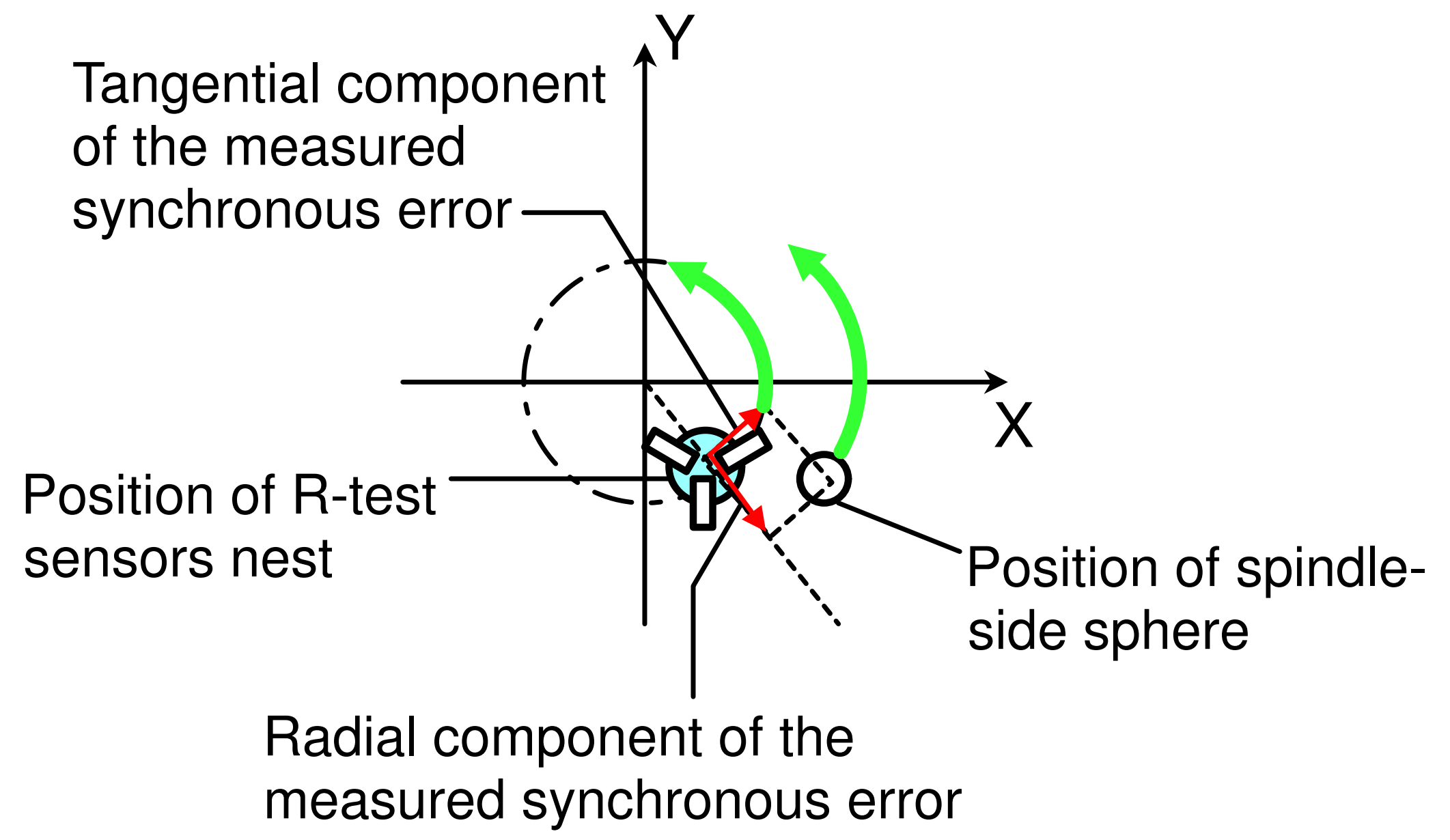




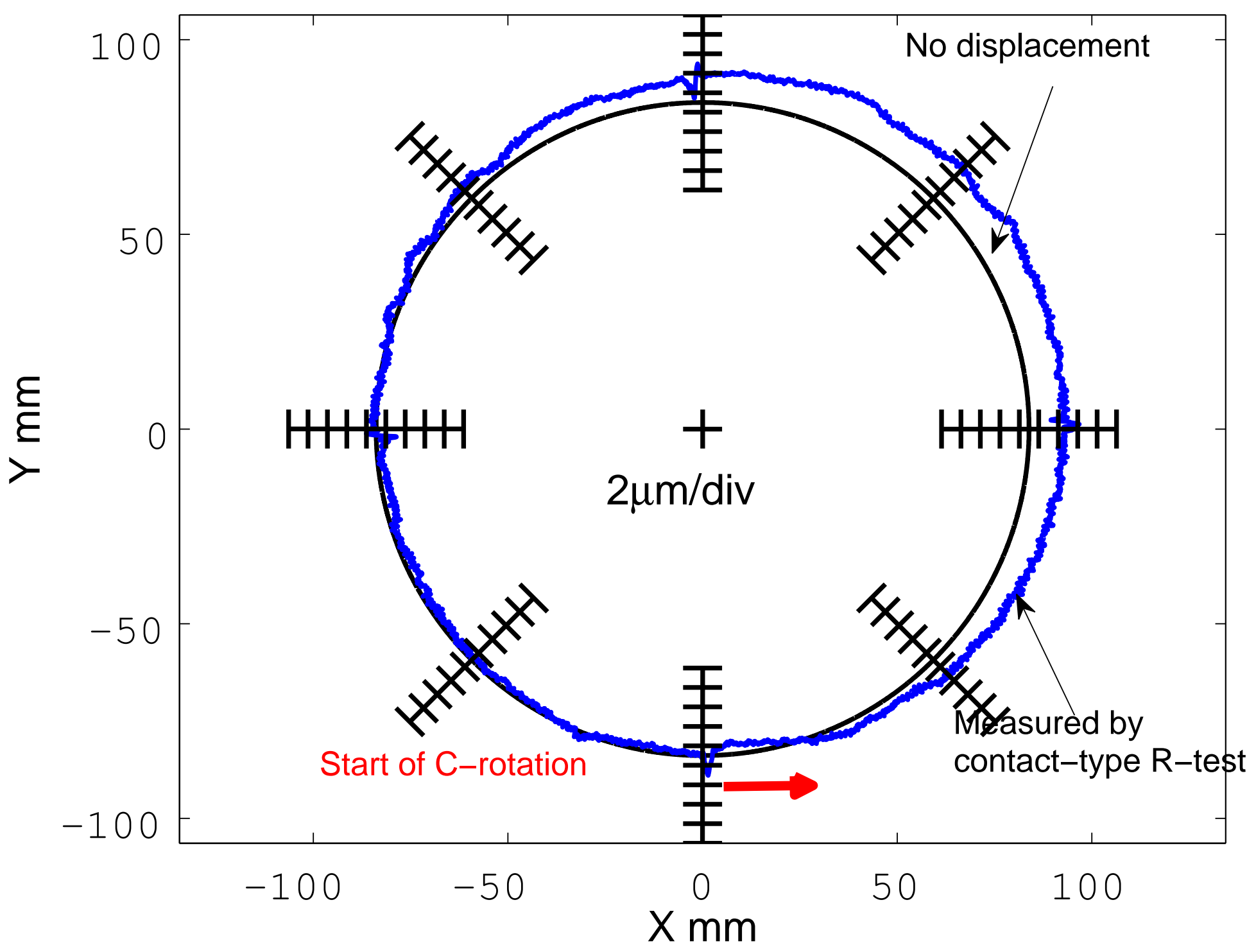




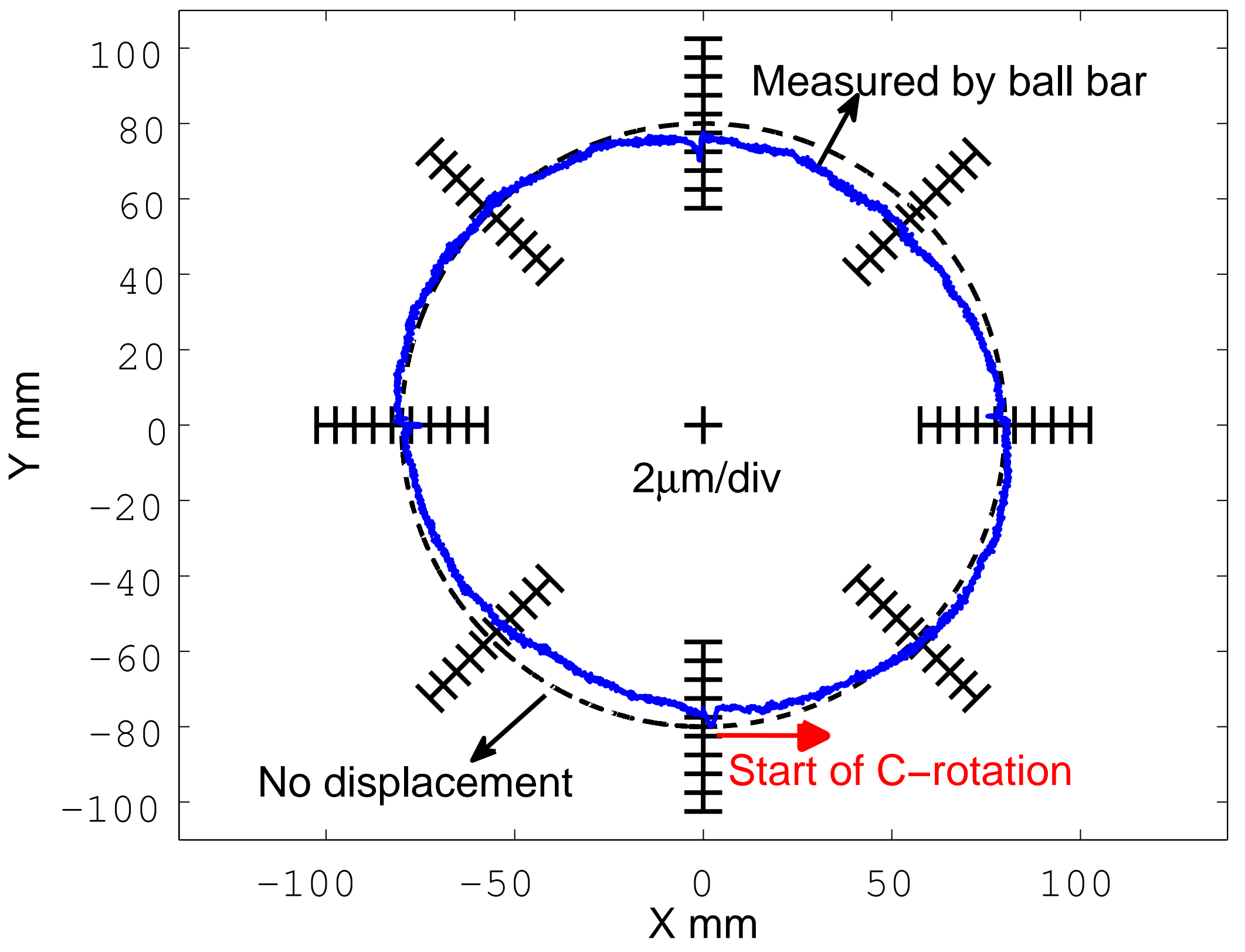




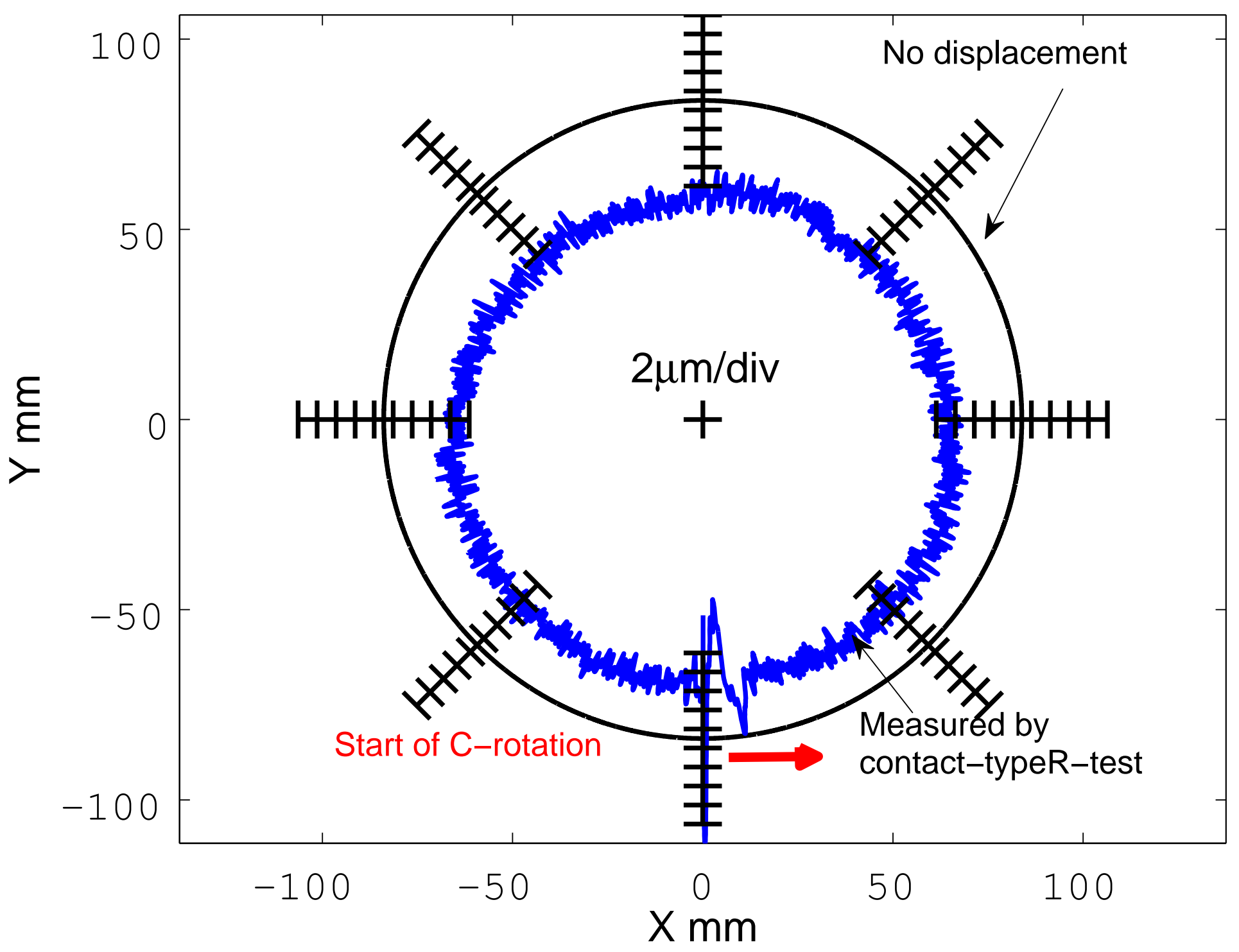




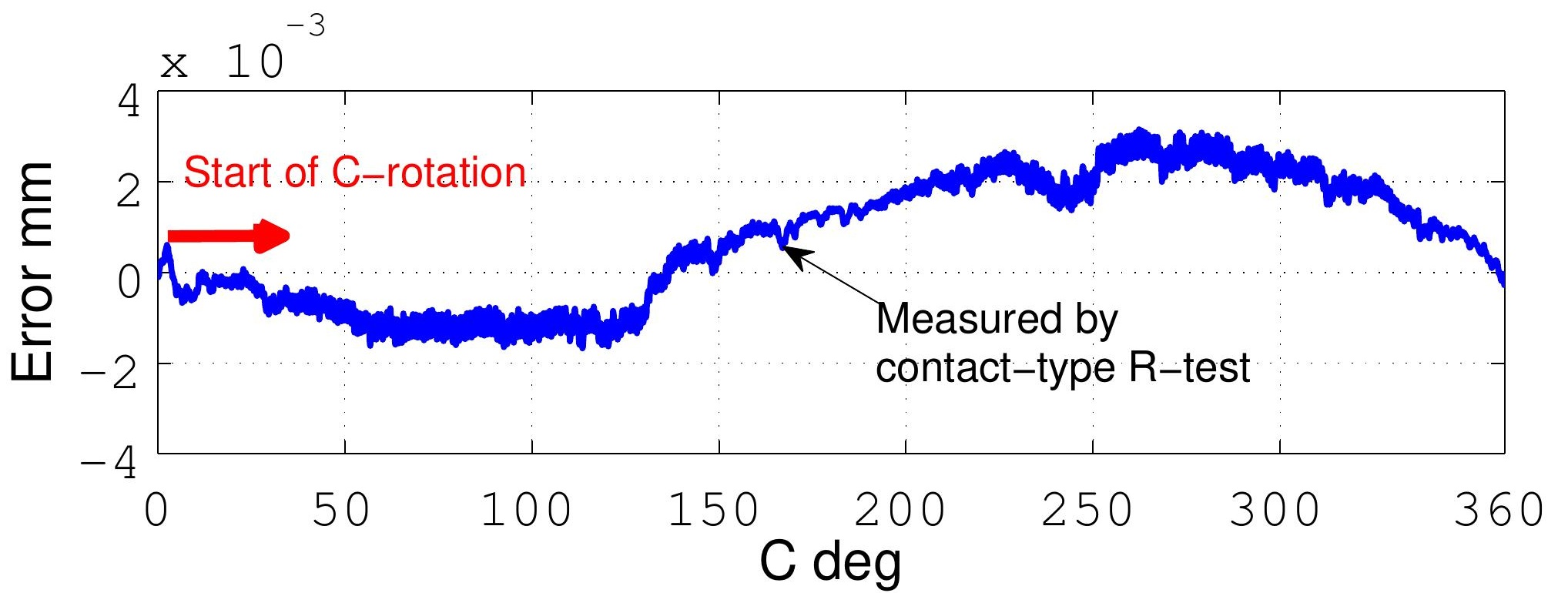




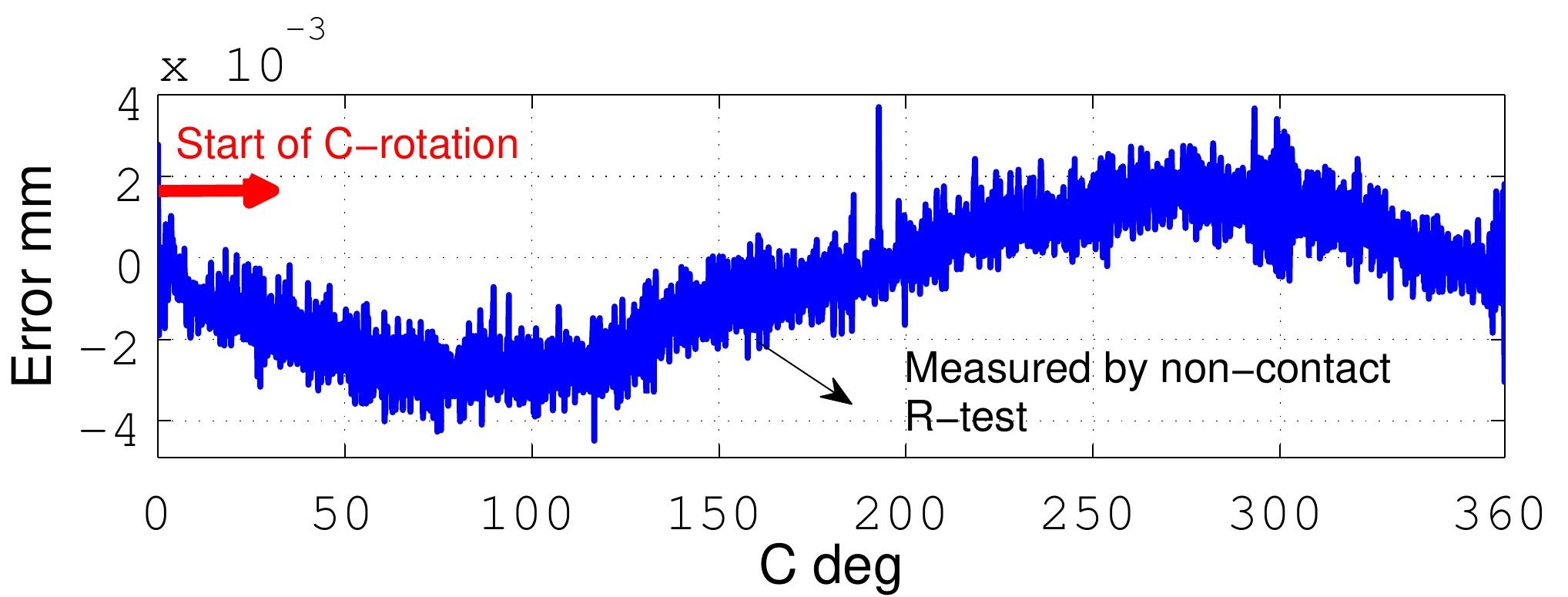




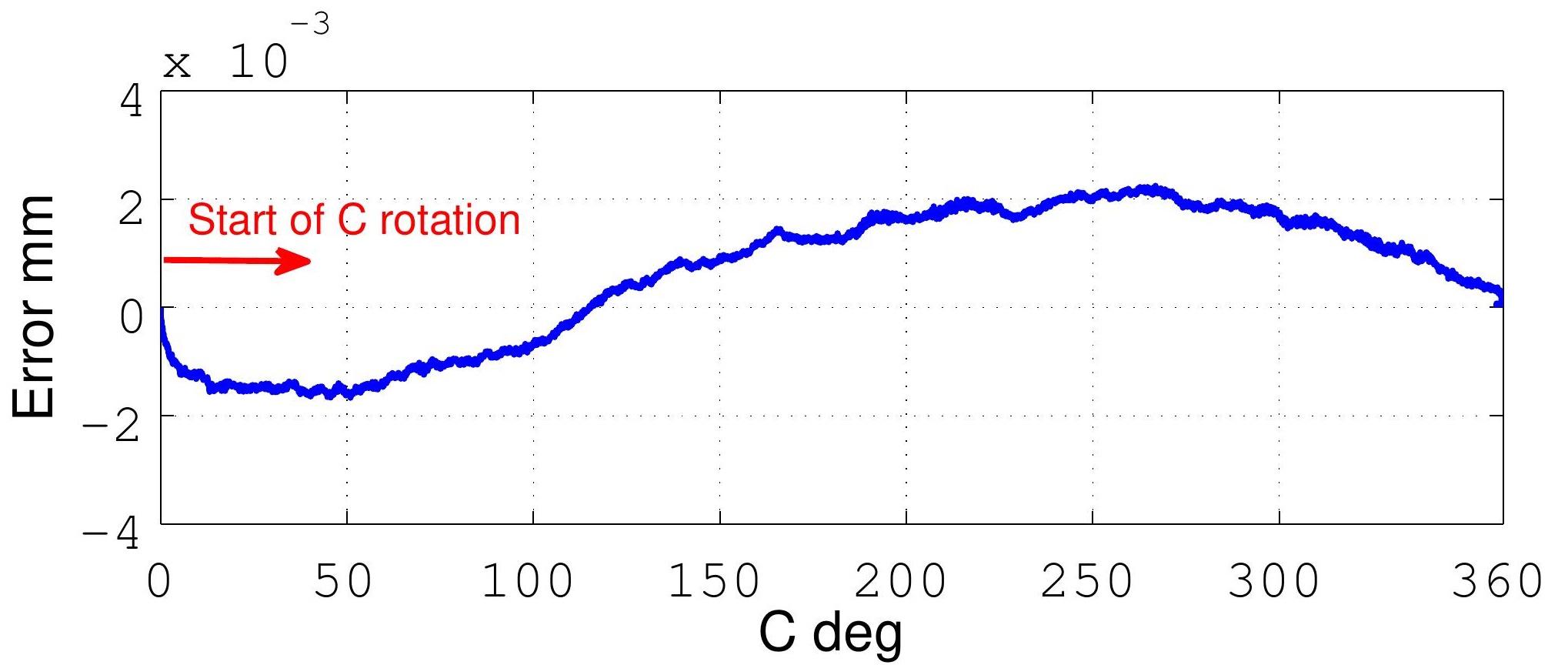




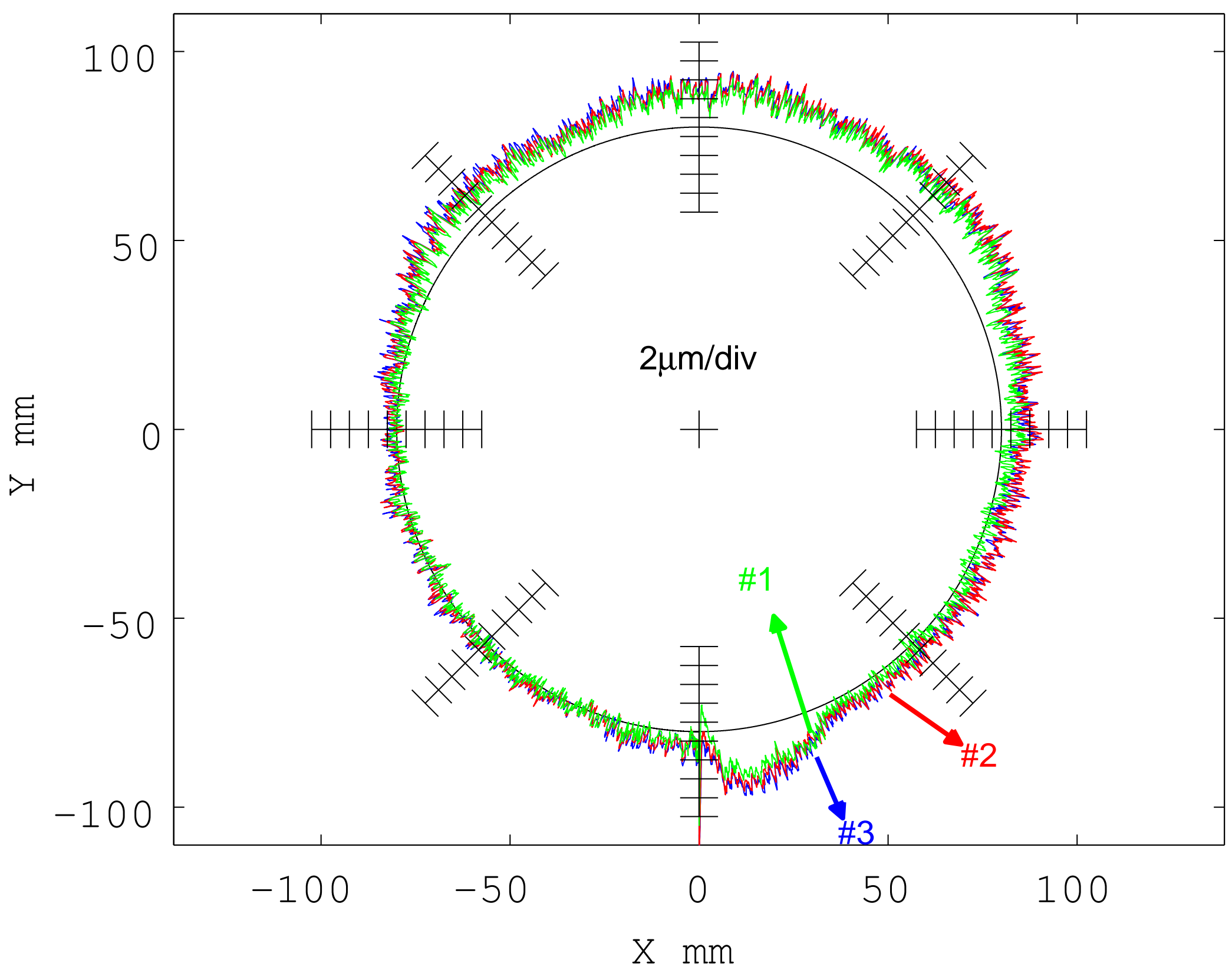


Table 1 Main specifications of laser displacement sensors used in this study [16]

\begin{tabular}{l|l|l|l|l}
\hline Maker: Keyence & LK-G10 & LK-H052 & SI-F10 & LT-9010MS \\
\hline Measuring principle & $\begin{array}{l}\text { Specular } \\
\text { reflection type }\end{array}$ & $\begin{array}{l}\text { Diffuse } \\
\text { reflection type }\end{array}$ & $\begin{array}{l}\text { Spectral } \\
\text { interference type }\end{array}$ & $\begin{array}{l}\text { Confocal } \\
\text { reflection type }\end{array}$ \\
\hline Reference distance & $10 \mathrm{~mm}$ & $50 \mathrm{~mm}$ & $11.3 \quad \mathrm{~mm}$ to & $6 \mathrm{~mm}$ \\
Measurement range & $\pm 1 \mathrm{~mm}$ & $\pm 10 \mathrm{~mm}$ & $12.35 \mathrm{~mm}$ & $\pm 0.3 \mathrm{~mm}$ \\
\hline $\begin{array}{l}\text { Spot diameter } \\
\text { (at reference distance) }\end{array}$ & $\Phi 20 \mu \mathrm{m}$ & $\Phi 50 \mu \mathrm{m}$ & $\Phi 40 \mu \mathrm{m}$ & $\Phi 2 \mu \mathrm{m}$ \\
\hline
\end{tabular}


Table 2 Specifications of the reference sphere (from Moritex Co., Ltd)

\begin{tabular}{c|c}
\hline Diameter & $25.4 \mathrm{~mm}( \pm 1.25 \mu \mathrm{m})$ \\
\hline Material & AL203 \\
\hline $\begin{array}{c}\text { Accuracy } \\
\text { grade }\end{array}$ & AFBMA Grade $5($ with \\
sphericity tolerance of $0.13 \mu \mathrm{m})$ \\
\hline
\end{tabular}


Table 3 Summary of profile measurement of a ceramic sphere (diameter:25.4 $\mathrm{mm})$

\begin{tabular}{c|l|l}
\hline Model & $\begin{array}{c}\text { Profile measurable } \\
\text { area }\end{array}$ & \multicolumn{1}{|c}{$\begin{array}{c}\text { Noise level } \\
(1 \sigma)\end{array}$} \\
\hline $\begin{array}{c}\text { Specular reflection type } \\
\text { (LK-G10) }\end{array}$ & $\begin{array}{c}1): \pm 0.3 \mathrm{~mm} \\
2): \pm 0.6 \mathrm{~mm}\end{array}$ & $1 \mu \mathrm{m}$ \\
\hline $\begin{array}{c}\text { Diffuse reflection type } \\
\text { (LK-H052) }\end{array}$ & $\begin{array}{l}1): \pm 1 \mathrm{~mm} \\
2): \text { over } \pm 2.5 \mathrm{~mm}\end{array}$ & $5 \mu \mathrm{m}$ \\
\hline $\begin{array}{c}\text { Spectral interference type } \\
\text { (SI-F10) }\end{array}$ & $\begin{array}{c}1): \pm 0.3 \mathrm{~mm} \\
2): \pm 0.3 \mathrm{~mm}\end{array}$ & $0.1 \mu \mathrm{m}$ \\
\hline $\begin{array}{c}\text { Confocal type } \\
\text { (LT-9010MS) }\end{array}$ & $\begin{array}{l}1): \pm 0.3 \mathrm{~mm} \\
2): \pm 1.5 \mathrm{~mm}\end{array}$ & $0.5 \mu \mathrm{m}$ \\
\hline
\end{tabular}


Table 4 Specifications of the reference sphere (from Kolb \& Baumann).

\begin{tabular}{c|c}
\hline Diameter & $50 \mathrm{~mm}+1.5 \mu \mathrm{m}$ at $20.6^{\circ} \mathrm{C}$ \\
\hline Sphericity & below $0.4 \mu \mathrm{m}$ \\
\hline Material & ceramic \\
\hline
\end{tabular}


Table 5 Specifications of the linear displacement sensor (MT1281 from Heidenhain).

\begin{tabular}{l|l}
\hline Measuring principle & $\begin{array}{l}\text { Photo-electric scanning of an incremental } \\
\text { scale with spring-tensioned plunger }\end{array}$ \\
\hline Measurement range & $12 \mathrm{~mm}$ \\
\hline System accuracy & $\pm 0.2 \mu \mathrm{m}$ \\
\hline Gauging force (vertically upward) & 0.35 to $0.6 \mathrm{~N}$ \\
\hline Signal period & $2 \mu \mathrm{m}$ \\
\hline Mechanically permissible traversing speed & $30 \mathrm{~m} / \mathrm{min}$ \\
\hline
\end{tabular}

